\title{
QUEEN'S
UNIVERSITY
BELFAST
}

\section{Load and harmonic distortion characterization of modern low-energy lighting under applied voltage variation}

\author{
McLorn, G., Laverty, D., Morrow, D., \& McLoone, S. (2019). Load and harmonic distortion characterization of \\ modern low-energy lighting under applied voltage variation. Electric Power Systems Research, 169, 124-138. \\ https://doi.org/10.1016/j.epsr.2018.12.029
}

\author{
Published in: \\ Electric Power Systems Research
}

\section{Document Version:}

Peer reviewed version

Queen's University Belfast - Research Portal:

Link to publication record in Queen's University Belfast Research Portal

\section{Publisher rights}

Copyright 2019 Elsevier.

This manuscript is distributed under a Creative Commons Attribution-NonCommercial-NoDerivs License

(https://creativecommons.org/licenses/by-nc-nd/4.0/), which permits distribution and reproduction for non-commercial purposes, provided the author and source are cited

\section{General rights}

Copyright for the publications made accessible via the Queen's University Belfast Research Portal is retained by the author(s) and / or other copyright owners and it is a condition of accessing these publications that users recognise and abide by the legal requirements associated with these rights.

Take down policy

The Research Portal is Queen's institutional repository that provides access to Queen's research output. Every effort has been made to ensure that content in the Research Portal does not infringe any person's rights, or applicable UK laws. If you discover content in the Research Portal that you believe breaches copyright or violates any law, please contact openaccess@qub.ac.uk. 


\title{
Load and Harmonic Distortion Characterization of Modern Low-Energy Lighting under Applied Voltage Variation
}

\author{
Gareth McLorn ${ }^{\mathrm{a}}$, David Laverty ${ }^{\mathrm{a}}$, D. John Morrow ${ }^{\mathrm{a}}$, Seán McLoone ${ }^{\mathrm{a}}$ \\ ${ }^{a}$ School of Electronics, Electrical Engineering and Computer Science, \\ Queen's University Belfast, Northern Ireland, U. K.
}

\begin{abstract}
This paper investigates the voltage level dependencies of different modern lighting types. Experiments are undertaken and the results used to formulate polynomial load models, presented in a ZIP format. These characterize active power, reactive power and harmonic current behaviors; are compatible with established power systems simulation practice; and are generally demonstrated to muster a good fit to the raw experimental data. Despite exceedingly high levels of current distortion being noted for several lamp instances, the attenuating effects of lowering voltage and harmonic diversity, as captured within a pair of diversity factor terms, are shown to help significantly alleviate the associated harmonic pollution impact on low voltage networks.

Keywords: load modeling, power quality, reactive power, total harmonic distortion, ZIP coefficients, harmonic diversity
\end{abstract}

\section{Introduction}

This paper investigates the non-linear characteristics of modern, commercially available Light Emitting Diode (LED) and Compact Fluorescent Lamp (CFL) luminaires, specifically how their behaviors vary with the applied voltage level of the electricity supply. Previous efforts to characterize these device types include the development of static load models in [1, 2]; the reporting of LED lamp attributes, including luminous efficacy and performance against ambient temperature, in [3]; and an assessment of the 
harmonic distortion footprint of LED lamps in [4], CFLs in [5] 9] and both technologies in [10, 11]. This paper seeks to further contribute to these themes by exploring the relationship between applied voltage level and the harmonic current distortion and power consumption characteristics of different luminaire types. Experimental results demonstrate that a strong correlation exists between these performance metrics.

The efforts of this paper are important in the context of the ongoing evolution of lighting technology, exemplified by developments such as the regulatory obliged demise of General Incandescent Lamps (GILs) [12]. The direct displacement of a major linear load type with non-linear alternatives [3, 9], such as CFLs [13] and latterly LED lamps (projected to capture $90 \%$ of the U.S. lighting market by 2035 [14]), is perceived to have altered typical end-user demand profiles [12].

The adaptation of a key constituent of feeder level demand has implications for utilities, especially in terms of load modeling for low voltage (LV) networks. Various research [5, 6] contends that the long-standing practice of aggregating various static load models to simulate large scale system interactions may no longer prove sufficiently accurate for networks in which substantive non-linear effects abound. Impacts upon the continued effectiveness of established network management and optimization techniques, such as Conservation Voltage Reduction (CVR), may also be considered [15]. This research contributes to the topic by arguing that static load models can be adapted to this evolved purpose, and thus retain their validity. An updated method of characterization for harmonic polluting devices is detailed, alongside a new approach for the modeling of harmonic current phasors.

Moreover, the typically low power classification of many modern lamps poses future power quality risks for utilities. The latest international standards [16] continue to prescribe relaxed harmonic current distortion limits for lamps rated between 5 and $25 \mathrm{~W}$. Despite their low power, the influence of many such devices may give rise to 
pronounced distortive effects within LV networks [9, 17]. However, their aggregated impact may be overstated, if the harmonic attenuating interactions among collections of lamps are not additionally factored. Thus, in the interests of analytical rigor, the concept of harmonic diversity ought to be explored [5, 6].

A novel experimental approach is hereby introduced, from which a set of load models are borne that encapsulate device behaviors in relation to fundamental powers and non-fundamental harmonic currents. In light of the ambient temperature and supply distortion induced variations observed for the currents drawn by appliances in [3] and [15], the testing methodology presented in this paper ensures that raw measurements are obtained in a manner that is sufficiently isolated from the influence of either factor. The deliberations of IEEE standard 1459-2010 [18] provide an impetus for excluding the presence of voltage distortion within such tests. On the assumption that practical network conditions apply and total harmonic current distortion $\left(\mathrm{THD}_{I}\right)$ levels exceed 40 $\%$, an apparent power orientated simplification is espoused in [18], such that the power quality test results are used to accurately characterize non-linear device behaviors.

An established, polynomial based characterization method is outlined, through which raw measurements are translated into a set of voltage response driven load models, for a selection of modern lighting types. A singular model for harmonic current phasors is hypothesized, through which voltage led fluctuations for both the magnitude and phase angle attributes are encapsulated. This is demonstrated to provide a suitable means through which multiple, harmonic polluting, load instances may be aggregated, in such a manner that the attendant cancellation effects [6, 11] of harmonic diversity are factored. Consolidated, lump load modeling of harmonic polluting devices is therefore made possible. This can contribute to the accurate representation of harmonic producing loads within load flow simulations. Moreover, the characterization parameters obtained from this study are conveniently tabulated for future reference. To assist in 
this endeavor, an angular metric for harmonic diversity is introduced and analyzed, alongside more established, current magnitude focused terminology. The remainder of the paper is organised as follows. \$2 reviews load modelling approaches and relevant practices and standards for power quality. \$3 describes the experimental procedure undertaken to capture the relationships between applied voltage level and the loading and harmonic current distortion attributes of selected luminaires. The influence of ambient temperature upon measured device characteristics is also considered. \$4 presents the experimental results, followed by analysis of the corresponding effects of harmonic diversity in $\$ 5$. A discussion of these results in relation to existing power quality standards and other published research [5, 6] is provided in \$6. Finally, conclusions on the impact that high penetrations of modern luminaires may have on future networks are presented in \$7, alongside some topics for further research.

\section{Harmonic Distortion and Associated Load Modeling Challenges}

Notable shifts have occurred in the load preferences of residential customers in re-

cent years. These include a proclivity for consumer electronics and the displacement of conventional forms of lighting with low energy alternatives [13, 19]. This is reflected within the increasingly non-linear nature of the power flows observed within LV networks [6]. In time, undesirable associated phenomena may foster new operational complications for utilities, such as growing levels of $T H D_{I}$ and voltage unbalance factor (VUF), and subsequent voltage quality effects therein [6, 9]. The ongoing validity of existing modeling practices, including the use of established static load models, ought to be scrutinized in this context.

\subsection{Load Modeling}

Several accepted static load modelling techniques exist, each of which characterizes the behavior of loads in response to steady-state variations in applied voltage and 
system frequency [20, 21]. Among the most popular methods is the ZIP polynomial model [21]. This is a quadratic function of applied voltage that is typically used to model the active, $P$, and reactive, $Q$, powers drawn by a load [1, 2, 15]. Its composition is presented in equations (1) and (2), where $P_{0}$ and $Q_{0}$ denote the active and reactive powers observed at nominal voltage, $V_{0}$. Further explanation of this model, and its three scalar coefficients, is provided in [15].

$$
\begin{aligned}
& P(V)=P_{0} \cdot\left[Z_{p} \cdot\left(\frac{V}{V_{0}}\right)^{2}+I_{p} \cdot\left(\frac{V}{V_{0}}\right)+P_{p}\right] \\
& Q(V)=Q_{0} \cdot\left[Z_{q} \cdot\left(\frac{V}{V_{0}}\right)^{2}+I_{q} \cdot\left(\frac{V}{V_{0}}\right)+P_{q}\right]
\end{aligned}
$$

By definition, the three ZIP coefficients in each equation must sum to 1. Many modern load types are observed to exhibit non-linear behavior, whereby changes to the load current are not proportional to fluctuations in applied voltage level. Such behaviors tend to be encapsulated by varying degrees of constant current $\left(I_{p}, I_{q}\right)$ and constant power $\left(P_{p}, P_{q}\right)$ attributes, which [21] discusses in greater detail.

\subsection{Power Quality Standards}

In the absence of corrective compensation, non-linear loads will, by definition, modulate the incoming supply voltage to draw a distorted current waveform. For those appliances that draw less than 16 A, IEC standard 61000-3-2 [16] stipulates performance criteria, including harmonic current limits. These are delineated into one of four classifications, A to D, with Class C incorporating all forms of lighting. The limits specified for Class $\mathrm{C}$ devices in [16] are reproduced in Table 1, where $\lambda$ denotes a device's displacement power factor $(\mathrm{DPF}){ }^{1}$.

\footnotetext{
${ }^{1}$ ratio of active to apparent power draw at fundamental frequency
} 
The limits set out in Table 1 are eased for lamps with active power ratings in the 5 to $25 \mathrm{~W}$ band. These low power adjusted limits, which allot to three different permissible performance frameworks [16], are displayed as percentages of the prevailing fundamental current in Table 2. The first set of requirements, group (i), is revised from a set of $\mathrm{mA} / \mathrm{W}$ specified limits, on the assumption that a nominal voltage of $230 \mathrm{~V}$ applies. Group (ii) combines comparatively loose magnitude limits with strict controls on current phase angle behaviors. Group (iii), is specified alongside an overall limit for $\mathrm{THD}_{I}$ of $70 \%$. The delineation of harmonic current pollution standards at $25 \mathrm{~W}$ is discussed in other work [4, 6, 10, 11, 15], although each pertains to the previous revision of IEC 61000-3-2 (2014 [22]), in which all non-discharge lighting types rated below 25 $\mathrm{W}$ were entirely exempted from harmonic current restrictions.

\subsection{Influence of Supply Voltage Distortion}

IEEE standard 1459-2010 [18] describes how the coupling of the distinct concerns of total harmonic voltage distortion $\left(\mathrm{THD}_{V}\right)$, pertaining to the electricity supply, and $T H D_{I}$, attributable to connected appliances, serve to magnify the deleterious effects of distortion across LV networks. As such, utilities in Europe are mandated, by the EN 50160 standard for voltage quality [23], to ensure that the ten-minute averaged levels of $T H D_{V}$ observed at customer points of common coupling (PCCs) are within $8 \%$.

The interaction of $T H D_{V}$ and $T H D_{I}$ is generally observed to increase the distortive content of the apparent power drawn by harmonic current inducing loads [15]. This quantity is defined as Non-Fundamental Apparent Power, denoted $S_{N}$. For reference,

further review of this quantity, and its derivation, is given in [15], from which it is shown that the ratio of $S_{N}$ to the fundamental component of apparent power, $S_{1}$, can be expressed, exclusively, as a function of $T H D_{V}$ and $T H D_{I}$ : 


$$
\frac{S_{N}}{S_{1}}=\sqrt{\left[T H D_{I}^{2}+T H D_{V}^{2}+\left(T H D_{I}^{2} \cdot T H D_{V}^{2}\right)\right]}
$$

In [15] and [24], different ZIP load modeling approaches are adopted to characterize the harmonic distortion ratios and non-fundamental apparent powers. For practical networks, $T H D_{I}$ levels are often much higher than $T H D_{V}$ and thus, as anticipated by (3), exert a more pervasive influence within the formation of harmonic power flows [15, 18]. Indeed, for conditions in which $T H D_{V}<5 \%$ and $T H D_{I}>40 \%$, it is argued that $S_{N} \simeq S_{1} \cdot T H D_{I}$ provides an acceptable approximation [18]. This tenet is validated in [15] where, given an expectation that $T H D_{V}$ shall not exceed $8 \%$ (in accordance with [23]), a set of ZIP models generated for $T H D_{I}$ are observed to provide accurate estimates for rms apparent power $(\mathrm{S})$, in the midst of applied voltage variations.

The observations of [15] demonstrate that desirable test conditions, for evaluating the harmonic ampere contributions of appliances and the complementary creation of voltage dependent, static load models, are best achieved by limiting $T H D_{V}$ to very low levels. This ensures that cross projections of $T H D_{V}$ and $T H D_{I}$ upon device performance, and those associated distortion power quantities for which $T H D_{V}$ is a factor [15, 18], are rendered negligible.

In effect, appliance behaviors, as observed under practicable conditions $\left(T H D_{V} \leq\right.$ $8 \%$ ), may reasonably be inferred (in terms of $S$ ) from $V_{1}$ dependent load models, alone.

This modelling proposition offers a reasonable approximation, but not an exhaustive characterization, of lamp behaviors under applied voltage variation (inclusive of a small degree of harmonic content). However, its limitations are acknowledged, especially the manner by which accuracy declines as $T H D_{V}$ levels rise [15], and so while its performance remains acceptable for most practicable instances of supply distortion [15], it is best suited for application at low, or zero, levels of $T H D_{V}$. To achieve a complete, voltage dependent characterization of non-linear device behaviors, especially 
for harmonic current distortion, additional models for $I_{h}\left(V_{n}\right), I_{h}\left(V_{n+1}\right) \ldots$ etc, and their cross projections, must be developed. The prospective undertaking of any such additional efforts, may consider the trade off that exists; the small potential increases in modeling accuracy versus the extra computational burden and unlimited number of potential harmonic and interharmonic interactions that may need to be quantified.

The findings of [15] are expanded upon, to propose a method for characterizing harmonic current by generating ZIP models for individual harmonic orders rather than the overall $T H D_{I}$ metric. The documented approach facilitates modeling of both the magnitude and phase angle properties of harmonic current, as functions of $V_{1}$.

\subsection{Harmonic Diversity}

The harmonic current signatures of different appliances diverge by magnitude and phase angle. Reasons for angular disparity include differences in system impedance, as observed at the PCC from the input terminals of a connected load [6]; the size of dc link capacitors [5] and loading conditions, taken with respect to device rated power [5].

For each harmonic current order, the spread of angles observed across multiple consumer devices gives rise to cancellation effects upon aggregation within adjoining LV networks. If a non-zero phase angle difference is observed between harmonic sources, the vector resultant of aggregate current shall be less than the equivalent arithmetic sum (of the respective harmonic magnitudes by device). This observation is summarized within a ratio term known as the magnitudinal harmonic diversity factor, $M D F_{h}[5$, 6. 11], for which lower values signify a greater spread of harmonic phase angles and consequentially, a more pervasive cancellation effect. In [10], the term phase angle diversity factor is used to describe the same effect. For a harmonic current of order $h$, $I_{h} \angle \phi_{h}$, the magnitudinal diversity factor pertaining to the aggregate current drawn by $N$ different loads is: 


$$
M D F_{h}=\left|\frac{\sum_{j=0}^{N} I_{h, j}}{\sum_{j=0}^{N}\left|I_{h, j}\right|}\right|
$$

Harmonic diversity is studied for some switch-mode power supply (SMPS) loads, including CFLs, in [5, 6, 10, 11], and LED lamps in [4, 10, 11]. In all cases, typical $M D F$ levels are observed to gradually decline as more devices connect in parallel. As $N$ increases further, an asymptotic level for $\overline{M D F}$ is attained, beyond which cancellation benefits diminish [5]. In general, harmonic phase angle disparity with CFLs is narrower than with LED lamps, for which the cancellation effect is more pronounced [10, 11].

An additional facet of this phenomenon is introduced and examined in this research, within a related metric termed the angular harmonic diversity factor, $A D F_{h}$. This ratio term aims to quantify the influence of harmonic cancellation upon the resultant phase angle of the aggregated harmonic current signal. For harmonic currents of order $h,\left|I_{h, j}\right|$ $\angle \phi_{h, j}$, the angular diversity factor pertaining to the amalgamated current drawn by $N$ different loads is defined in (5). The numerator denotes the angle of the resultant current phasor (scaled by $N$ ), with the denominator indicating the maximum possible angular swing that may be exerted, for currents of order $h$, as a consequence of aggregation. The introduction of this factor builds upon the studies in [25], where the harmonic current phase angles of various aggregate load mixes are shown to converge to a fixed value, for group sizes of thirty and over.

$$
A D F_{h}=\frac{N \cdot \operatorname{Arg}\left(\sum_{j=0}^{N} I_{h, j}\right)}{\sum_{j=0}^{N}\left|\operatorname{Arg}\left(I_{h, j}\right)\right|}
$$

This theme is explored in the wider context of aggregate load modelling to encompass an extensive selection of electrical appliance types. In [25-27], a single, composite harmonic current footprint is resolved from the attributes of individual devices to form a consolidated lumped load model, also referred to as an aggregate harmonic load (AHL) 
in [26]. In [25 27], ADLs are continuously updated, according to various load mixes and so are configured to reflect the time-varying nature of demand trends within practical networks. The creation of time series profiles for harmonic current is subsequently made possible, although in the surveyed literature this is only enacted for harmonic current magnitudes, rather than phase angle data. AHLs can be defined at various levels of network abstraction [25], such as from the perspective of a single household [27] or to characterise the entire secondary LV network served by a step-down transformer [26, 28]. This approach can help simplify the simulation of very large network models, in which detailed analysis of all LV sub-circuits may not be required [29].

In some literature, the non-linear constituent of AHLs are composed from the amalgamation of equivalent circuit models rather than polynomial based models [27, 28]. This method is based on prior research performed in [5], for which an inherent weakness is acknowledged in terms of its application to the aggregation of harmonic behaviors within the lumped SMPS models produced. This pertains to the "ability to correctly model harmonic cancellation between the individual SMPS loads" owing to the absence of consideration for harmonic phase angles within the constituent load circuit models. In [26], this limitation is countered via the probabilistic assignment of a synthetic magnitudinal diversity factor, which is used to scale the sum of harmonic current magnitudes to an appropriately attenuated aggregate level.

Conversely, the polynomial based load modelling technique, espoused for harmonic current characterization in this paper, does not require the additional synthesis of $M D F_{h}$ values. The natural harmonic cancellation effect is implicit within the method, such that the resolution of (4) with the ZIP model estimates is proven to be consistent with real world observations. Moreover, previous research has neglected the influence of supply voltage magnitude diversity across networks, in invoking a heightened sense of phase angle diversity between load instances, and thus a more pervasive cancellation 
effect. This shortcoming is remedied within the models proposed by this paper.

\section{Methodology}

\subsection{Apparatus}

An overview of the test equipment is given in Fig. 1. It includes a relay test and calibration tool, which acts as a high-fidelity voltage source for the device-under-test (DUT), ensuring that the potentially degrading influence of supply voltage distortion on the results is effectively eliminated. This unit feeds a power meter, which serves as both a protective buffer for the signal source and a measurement verification aid for the experiment. This feeds power to the single-phase connected DUT and provides voltage monitoring access to power quality data logging apparatus. Current is sensed as it flows along the neutral return wire from the DUT to the power meter. Fundamental and rms current; active, reactive and non-fundamental power; power factor; and the magnitudes and phases angles of non-fundamental, harmonic voltages and currents; are logged at one second intervals. The logging apparatus also quantifies $T H D_{I}$ results through the accumulation of measurements for each harmonic current order, up to $h=40$.

\subsection{Experimental Procedure}

To obtain a valid ZIP load representation of a luminaire, the impact of ambient temperature on its performance must be isolated from that of voltage, as discussed in [3]. One means of mitigation is to minimise the temporal variation in temperature observed across each experiment, as the applied voltage is adjusted to different levels. This is achieved by monitoring temperature within the DUT enclosure using a thermocouple

device. A testing method approved by the Illuminating Engineering Society, IES LM79-08 [30], was chosen from which an appropriate temperature range of $25 \pm 1^{\circ} \mathrm{C}$ was defined for application within each experiment. 
Experiments begin by powering on the selected DUT at nominal voltage until the enclosure warms beyond $24^{\circ} \mathrm{C}[30]$. Each voltage level applied to the DUT is maintained for a period of two minutes; during which power quality data is logged on a per second basis, while temperature readings are recorded at one-minute intervals.

For LED modules the applied voltage is increased in steps of $0.02 \mathrm{pu}$ across the statutory range (0.90 - $1.10 \mathrm{pu}[23])$. Additional care is taken while testing CFLs. For each voltage increment, the ensuing power transitions are observed to take roughly twenty minutes to subside. Consequently, a delay of thirty minutes is applied after each new voltage level is asserted, prior to the logging of performance data. This approach is similar to [4], in which ten-minute stabilization delays are applied during testing. A larger voltage interval of $0.05 \mathrm{pu}$ is applied between readings for CFL testing, so that both the variation in temperature observed across the full body of readings and the subsequent effects upon ZIP load characterization are minimized.

In [4], adjustments to voltage are applied according to the SEMI F47 standard curve for voltage sag immunity testing [31]. This aspect contrasts to the linear ramping of voltage undertaken for this paper, across the statutory range.

\subsection{Formulation of ZIP coefficients from Experimental Data}

The ZIP coefficients are determined via least squares regression. Considering the

ZIP model for active power (1), the process begins by computing the least squares fit of the quadratic function

$$
\hat{P}=\theta_{1} V^{2}+\theta_{2} V+\theta_{3}
$$

to the measured P-V data. By denoting the $n$-length vector of power measurements as $\boldsymbol{y}=\left[\begin{array}{lll}P_{1} & \ldots & P_{n}\end{array}\right]^{T}$, constructing matrix $\boldsymbol{X}$ from the corresponding voltages as 


$$
\boldsymbol{X}=\left[\begin{array}{ccc}
V_{1}^{2} & V_{1} & 1 \\
\vdots & \vdots & \vdots \\
V_{n}^{2} & V_{n} & 1
\end{array}\right]
$$

and defining the vector of ZIP load coefficients as $\theta=\left[\begin{array}{ll}\theta_{1} & \theta_{2} \\ \theta_{3}\end{array}\right]^{T}$, the quadratic function can be expressed in matrix vector form as $\hat{\boldsymbol{y}}=\boldsymbol{X} \theta$. The least squares fit to the true data $\boldsymbol{y}$ is then obtained using the Moore-Penrose pseudo inverse approach:

$$
\theta=\left(\mathbf{X}^{T} \mathbf{X}\right)^{-1} \mathbf{X}^{T} \mathbf{y}
$$

Having computed $\theta$ in (8), the fitted value for $P_{0}$ is obtained from the solution for (6) in which $\mathrm{V}$ is set equal to $V_{0}$. ZIP parameters $Z_{p}, I_{p}$ and $P_{p}$, are then given by:

$$
\left[\begin{array}{c}
Z_{p} \\
I_{p} \\
P_{p}
\end{array}\right]=\frac{1}{P_{0}}\left[\begin{array}{ccc}
V_{0}^{2} & 0 & 0 \\
0 & V_{0} & 0 \\
0 & 0 & 1
\end{array}\right]\left[\begin{array}{l}
\theta_{1} \\
\theta_{2} \\
\theta_{3}
\end{array}\right]
$$

An advantage of this approach is that the $Z_{p}+I_{p}+P_{p}=1$ condition is automatically satisfied without further augmentation of the least squares problem. To engender confidence in the reported values, the final ZIP coefficients are specified alongside the coefficient of determination $\left(\mathrm{R}^{2}\right)$ that arises from the direct comparison made of the relevant $\mathbf{y}$ and $\hat{\mathbf{y}}$ data sets, as observed across the same set of applied voltage samples.

The methodology for obtaining $R^{2}$ from the experimental datasets that pertain to each tested lamp is described in the following. First, the regression sum of squares, $S S_{r e g}$, metric is formed, via (10). This compares the predicted values with the mean of the data set. 


$$
S S_{\text {reg }}=\sum_{j=0}^{N}\left(\widehat{y_{j}}-\bar{y}\right)^{2}
$$

Second, the residual sum of squares, $S S_{\text {res }}$, is calculated from (11), to quantify the prediction error between the raw and modeled data, whereby an error array is created via: $e_{j}=y_{j}-\widehat{y_{j}}$.

$$
S S_{r e s}=\sum_{j=0}^{N} e_{j}^{2}
$$

Finally, $R^{2}$ is given by the quotient:

$$
R^{2}=\frac{S S_{r e g}}{S S_{r e g}+S S_{r e s}}
$$

\subsection{Characterization of Harmonic Current Draw as a function of Applied Voltage}

The use of ZIP models to characterize voltage derived fluctuations in $P$ and $Q$ is common electrical engineering practice. This approach can be expanded to define similar model parameters for $T H D_{I}$ [15] or the individual harmonic current components drawn by the tested lamps. Given that ZIP polynomials are identified as being "without physical correspondence" [20], it is acceptable to frame the relationship between harmonic currents and applied voltage in this manner.

The magnitude and angle properties of each harmonic current order, $h$, are often presented as a singular polar variable. However, the associated ZIP characterization is expressed more conveniently in cartesian form; consisting of two orthogonal variables, $a_{h}$ and $b_{h}$. These pertain to two distinct load models; each expressed as a quadratic function of $V_{1}$ and $h^{t h}$ order specific sets of ZIP coefficients, such as $\theta_{h a}$ for $a_{h}$

and $\theta_{h b}$ for $b_{h}$

$$
\widehat{a_{h}}\left(V_{1}\right)=a_{0, h} \cdot\left[\theta_{h a, 1} V_{1}^{2}+\theta_{h a, 2} V_{1}+\theta_{h a, 3}\right]
$$




$$
\widehat{b_{h}}\left(V_{1}\right)=b_{0, h} \cdot\left[\theta_{h b, 1} V_{1}^{2}+\theta_{h b, 2} V_{1}+\theta_{h b, 3}\right]
$$

where $a_{h}=\left|I_{h}\right| \cdot \cos \left(\phi_{h}\right)$ and $b_{h}=\left|I_{h}\right| \cdot \sin \left(\phi_{h}\right)$, with the terms $a_{0, h}$ and $b_{0, h}$ being used to signify the respective values observed at nominal voltage, $V_{0}$.

The corresponding singular load model for each order is presented in (15). Similarly, the ZIP models expressed in (1)-(2) for $P$ and $Q$ are an extension of (15), where $h=1$ and the current phasor, $\left|I_{1}\right| \angle \phi_{1}$, is scaled by reference voltage phasor, $V_{1}$.

$$
\left|\widehat{I_{h}}\left(V_{1}\right)\right| \angle \widehat{\phi_{h}}\left(V_{1}\right)=\widehat{a_{h}}\left(V_{1}\right)+j \widehat{b_{h}}\left(V_{1}\right)
$$

To aid comprehension of the stated method, voltage led variations in the harmonic currents drawn by one tested lamp (Bulb 05, introduced in \$4) are interpreted via polar plots. Similar portrayals of harmonic currents are found in [4, 5, 10, 25, 29]. In Fig. 2 , current phasors of the six lowest, odd, non-fundamental orders, plus the fundamental, are illustrated in absolute mA terms. Even harmonic orders are discounted from the analysis. This adheres with a convention for power systems modelling in the steady state [32], in which symmetry between the positive and negative half-wave cycles of voltage and current signals is always assumed. The stated selection of non-fundamental, harmonic orders is also reasoned to contribute an equitable spread of negative (orders 5 and 11), positive (orders 7 and 13) and zero (orders 3 and 9) sequence effects [32], within any prospective, harmonic load flow study that may seek to incorporate the load models expounded by this research. Moreover, for each of the eleven tested lamps, the bulk of the recorded $T H D_{I}$ measurements is observed to accumulate within the six chosen harmonic current orders.

The values displayed in Fig. 2 are determined from the ZIP model characterization of experimental measurements for Bulb 05. The logged levels of $T H D_{I}$, pertaining to these polar plots, are observed to decline with applied voltage, from $145 \%$ at $1.10 \mathrm{pu}$ 
to $123 \%$ at $0.90 \mathrm{pu}$. The circular perimeter of each plot denotes a reference current of $50 \mathrm{~mA}$. Despite the reduction in $T H D_{I}$, in percentage terms, the attendant ampere magnitude of fundamental current is shown to grow with decreasing voltage in Fig. $2(\mathrm{~g})$, such that an overall increase in rms current ${ }^{2}$ is observed, a reflection of the constant power nature of the device. The harmonic phase angle associated with the fundamental corresponds to the displacement power factor angle, $\phi$. Fig. 2(g) shows how, for Bulb 05, $\phi$ increases and thus DPF (the cosine of $\phi$ ) reduces as the voltage level declines. All other non-fundamental phasors are observed to rotate by varying angular amounts as the $V_{1}$ level is adjusted.

\section{Experimental Results}

Details of the evaluated lamps are given in Table 3 . These include a $60 \mathrm{~W}$ rated GIL (Bulb 11) that chiefly serves as a performance reference for the CFL and LED lamps. The active and reactive power responses of each lamp are plotted against voltage level in Fig. 3 and Fig. 4. The reactive power contribution of the GIL is deemed negligible when compared to other bulbs, and so is omitted from Fig. 4 .

Nominal levels of active and reactive power, $P_{0}$ and $Q_{0}$, are specified as those values observed at $1.0 \mathrm{pu}$ voltage. As such, each of the per-unit characteristics depicted in Fig. 3 and Fig. 4 are shown to pass through the $(1,1)$ co-ordinate. Actual measured data is depicted by the scattered points, while full line trends chart the corresponding ZIP load estimates. Ambient temperature was successfully kept within the bounds recommended by [30] throughout. Table 3 also includes the ZIP coefficients determined for all evaluated lamps. The values reported for $\mathrm{Q}_{0}$ show that the reactive power contributions of all lamps are negative, and thus capacitive in nature.

For all CFLs, $\left|I_{1}\right|$ is recorded as decreasing with lowering voltage, while for all LED

${ }^{2} I=I_{1} \cdot \sqrt{T H D_{I}^{2}+1}$ 
lamps it is noted to rise. The DPF of all CFLs and LED lamps 01, 02 and 05 degrades with reducing voltage, but is shown to improve for LED lamps 03, 04, 06 and 07.

Tables 4 to 9 each present two sets of ZIP coefficients, determined for the cartesian form of non-fundamental harmonic currents $I_{3}$ to $I_{13}$ (odd orders only), across all evaluated lamps. Variables $a_{0, h}$ and $b_{0, h}$ are analogous to that of $P_{0}$ and $Q_{0}$ in Table 3. and pertain to the nominal magnitudes of $a_{h}$ and $b_{h}$, for a given harmonic order $h$. The polar form of $I_{h}$, expressed in percentage terms of $I_{1}$, is resolved within the two furthest right-hand columns of each table.

Regarding $I_{3}$ (Table 4), across all tested CFLs, GILs and LED lamp 07, lowering voltage proves effective in reducing $\left|I_{3}\right|$. For LED lamps 02, 03, 04 and $05,\left|I_{3}\right|$ is observed to increase with declining voltage. For the other LED lamps (01 and 06), reductions in voltage have a negligible effect. The contrasting influence of voltage reduction on $\left|I_{3}\right|$ is charted in $\mathrm{mA}$ terms for (a) LED lamp 02 and (b) CFL lamp 09 in Fig. 5. As applied voltage reduces from $1.10 \mathrm{pu}$ to $0.90 \mathrm{pu}$, a $19.7 \%$ increase in $\left|I_{3}\right|$ is noted for the former, while a $4.7 \%$ reduction is observed for the CFL.

Table 5 reveals that for all tested CFL, GILs and LED lamps 01, 03, 04, 06 and 07, lowering voltage is shown to be effective in reducing levels of $\left|I_{5}\right|$. However, for LED lamps 02 and $05,\left|I_{5}\right|$ is observed to increase (in ampere terms) as voltage decreases. Conversely, lower voltages are shown to be effective in reducing $\left|I_{7}\right|$ for all lamps. Apart from LED lamp 06 and CFL 08, $\left|I_{9}\right|$ is observed to decline as voltage is reduced (Table 7). Likewise, general decreases in $\left|I_{11}\right|$ are observed upon the application of voltage reduction, with exceptions noted for LED lamps 03, 05 and 07 (Table 8). Increases in $\left|I_{13}\right|$, with decreasing voltage, are observed for LED lamps 02, 05 and 07 (Table 9).

In the interests of validation, $R^{2}$ values are also reported within each set of results in Tables 3 to 9 . The vast number of these tend towards the ideal value of 1.00 , and so indicate the success of the ZIP models in engendering high quality fits against the 
underlying raw data. However, a small number of low $R^{2}$ instances are also present, which exposes the limitations of both the precision of the measurement equipment and the analytical approach, encapsulated by equations (1), (2), (13) and (14).

Disparities between the $R^{2}$ values obtained among cartesian paired terms, $\widehat{a_{h}}$ and

$\widehat{b_{h}}$, are noted for several harmonic current phasors in Tables 4 to 9 . This phenomenon is scrutinized in greater depth for LED lamp 04 (and phasor $I_{5}$ ), LED lamp 07 (and phasors $I_{3}$ and $I_{9}$ ) in Figs. 6, 7 and 8, respectively. These charts highlight how the presence of a poor/good fit combination within a cartesian pair translates into a similar discrepancy among the corresponding polar terms, $\left|I_{h}\right|$ and $\phi_{h}$.

\section{Harmonic Diversity}

In [6], the aggregated harmonic distortion impact of CFLs within LV networks is explored using a Monte Carlo approach. Network impedance, integrated dc link capacitance and fluorescent tube power are varied in simulation and the ensuing harmonic cancellation effect is analyzed. A similar method is adopted in this paper to investigate how fluctuations in applied voltage influence the extent of harmonic cancellation observed for groups of CFLs and LED lamps. In a practical sense, such analysis may prove useful in the context of an LV distribution circuit, along which an assortment of customers are connected. Despite the influence of system impedance on the harmonic diversity effect not being explicitly analyzed within this research, it is known from other literature [5, 6, 25] that the attenuation of harmonic current distortion among aggregated loads is expected to be more pervasive as system impedance increases, especially in the case of higher order harmonics. This finding is particularly pertinent within less stiff regions of the grid [5], such as radial sections of the distribution network.

In the first study, $N$ instances of a ZIP model pertaining to a specific lamp type are each subjected to a randomized applied voltage between 0.90 to $1.10 \mathrm{pu}$. The 
harmonic currents from the homogeneous, $N$ lamp grouping are summed and the vector and arithmetic resultants used to generate diversity factors for each harmonic order, $M D F_{h}$ and $A D F_{h}$, as per (4) and (5). For each order, $h$, this step is replicated for one thousand different randomized voltage permutations, from which mean diversity factors, $\overline{M D F_{h}}$ and $\overline{A D F_{h}}$, are computed. This process is repeated across different group sizes, whereby $N$ is increased progressively from 2 to 100 , and for each lamp type analyzed in this paper. Due to lamp type being common across each $N$-sized grouping, the $\overline{M D F}$ values reflect the magnitudinal harmonic cancellation potential due to a single variable, voltage variation. Annotated plots of the cancellation impact attributable to voltage variation are presented in Fig. 9(a) for all seven LED lamps and Fig. 9(b) for the three CFLs.

In Fig. 9, diversity led attenuation appears to be more prevalent within homogeneous groups of LED lamps than for CFLs. More generally, the cancellation impact is observed to be more pronounced for higher harmonic orders, with a negligible influence observed for the third and fifth orders. Moreover, as is the case in [6], the diversity effect grows for further increments of $N$, reaching asymptotic levels for $\overline{M D F_{h}}$ in Fig. 9 once group size reaches 50 lamps or more.

The second study is conducted on a similar basis. However, each $N$-sized grouping is composed from a mixed combination of either LED or CFL lamp type instances, rather than a homogeneous collection. Each combination is chosen at random, within which multiple instances of one lamp type may appear. Given the increased degrees of freedom, the sample size for each Monte Carlo experiment is raised to ten thousand. The resultant $\overline{M D F_{h}}$ and $\overline{A D F_{h}}$ values reflect the combined harmonic cancellation potential of voltage variability and technological diversity. Plots of the magnitudinal cancellation impact are presented in Fig. 10(a) for LED lamps and Fig. 10(b) for CFLs, while the similarly asymptotic nature of the angular harmonic diversity factor 
is observed in Fig. 11(a) for the same mix of LED lamps and Fig. 11(b) for CFLs.

In general, the magnitudinal diversity factors recorded across a mix of bulbs are much lower than those observed for multiple instances of the same bulb and so collections of this nature exhibit higher degrees of harmonic attenuation. The cancellation potential for multiple LED lamps appears to be more pervasive than with CFLs, as anticipated by [10, 11]. Once again, the attenuation of higher order harmonics is more substantial, although significant reductions in the third and fifth harmonics are noted among the collection of LED lamps (a converged $\overline{M D F_{h}}$ level of around 0.75 ).

These results show how the theory of harmonic diversity is inherently factored within the voltage driven, harmonic current phasor model introduced in 15 to create an holistic expression for fluctuations in aggregate harmonic currents on LV networks, as a function of the individual PCC voltages. This contrasts with the equivalent circuit based approach for non-linear load modelling in [5], in which harmonic cancellation is not apparent within the corresponding aggregate models. In this paper, for a sufficiently large group size, the expected magnitudinal diversity factor (4), $\overline{M D F_{h}}$, pertaining to harmonic current order, $h$, is demonstrated by Figs. 9 and 10 to tend towards an asymptotic level. This outcome echoes the findings of [25], in which convergence is observed for the third and fifth harmonic current orders for $N>30$. Moreover, the $M D F_{h}$ values reported for the practical investigation of CFLs and LED lamps in [1] are similar to those presented in this paper.

For circumstances in which harmonic phase angle data is not available but the expected value of $M D F_{h}$ is known, an expectation of the attenuated magnitude of the aggregate harmonic current, attributable to a collection of multiple lamp instances, may be determined from the sum of the expected individual phasor magnitudes.

$$
E\left[\left|\sum_{j=0}^{N} \widehat{I_{h, j}}\left(V_{1, j}\right)\right|\right]=\overline{M D F_{h}} \cdot \sum_{j=0}^{N} E\left[\left|\widehat{I_{h, j}}\left(V_{1, j}\right)\right|\right]
$$


Similarly, for large group sizes the asymptotic value of the mean angular harmonic diversity factor (5), $\overline{A D F_{h}}$, may be used to recover the expected phase angle of the aggregated harmonic current phasor, from the sum of the absolute values taken for each of the expected individual harmonic phase angles.

$$
E\left[\operatorname{Arg}\left(\sum_{j=0}^{N} \widehat{I_{h, j}}\left(V_{1, j}\right)\right)\right]=\frac{1}{N} \cdot \overline{A D F_{h}} \cdot \sum_{j=0}^{N} E\left[\left|\operatorname{Arg}\left(\widehat{I_{h, j}}\left(V_{1, j}\right)\right)\right|\right]
$$

\section{Discussion}

Fig. 3 shows how the active power consumption of modern lamps is consistently less responsive to changes in voltage than legacy types, i.e. GILs. For LED lamps, this mostly manifests in close to constant power behavior. By contrast, the active power drawn by CFLs is shown to be more susceptible to changes in voltage. The values reported for $Q_{0}$ in Table 3 highlight that the reactive power contributions of all tested lamps is nominally capacitive in nature. In Fig. 4, the reactive power of five of the tested LED lamps is observed to correlate positively with voltage, while for two bulbs (02 and 05) it is negatively correlated, rising in magnitude as voltage is reduced. For each CFL tested, reactive power is shown to gradually decline with decreases in voltage.

Results for nominal harmonic current magnitudes, denoted $\left|I_{0, h}\right|$ in Tables 4 to 9 , allude to significant levels of distortion for most LED lamps. For LED bulbs 01, 02 and 05, the nominal magnitudes of the third (Table 4), fifth (Table 5) and seventh (Table 6) harmonics exceed all the corresponding choices of requirements defined for lighting in Table 2, but would not have violated the previous, 2014 revision of IEC 61000-3-2 [22], given its sub-25 W exemption for non-discharge lighting types [15]. While $\left|I_{0,3}\right|$ and $\left|I_{0,5}\right|$ emissions from CFLs (08, 09 and 10) are substantial, they adhere with the group (ii) set of limits in Table 2. Marginal excursions of the seventh harmonic limit are noted for CFLs 09 and 10 in Table 6. However, given the prospective cancellation 
effect of lamp aggregation observed for these two devices (Fig. 10), the cumulative impact of connecting numerous CFLs to LV networks may prove to satisfy each of the requirements options in Table 2 , if one considers average bulb performance.

Nominal operation of LED lamps 01, 02 and 05 and all three CFLs (08, 09 and 10), at $230 \mathrm{~V}$, transgresses beyond the group (i) magnitude limits defined for both the ninth (Table 7) and eleventh (Table 8) harmonic currents in Table 2, Likewise, the limit for the thirteenth harmonic $(6.82 \%$; Table 2 , group $(i))$ is exceeded for each of these six lamps, plus LED lamp 06 (Table 9). LED lamp 03 is consistently shown to exhibit low levels of harmonic current distortion across all orders. This device is thought likely to incorporate active filtering circuitry, similar to that discussed in [5].

The influence of voltage reduction on $T H D_{I}$ is more difficult to quantify than for $P$ and $Q$, given its impact on both the magnitude and angle of harmonic current phasors. Per-unit variations in total non-fundamental harmonic current, $I_{H}$, are plotted against applied voltage, within Fig. 12 for each tested lamp. This chart shows that every tested CFL emits lower harmonic currents at lower voltage levels, as do all LED lamps with the exception of bulbs 02 and 05 . In general, one may conclude that voltage management strategies, such as CVR, may be expected to help suppress harmonic current emissions attributable to lighting, through the practice of lowering of PCC voltages.

In $\S 4$, the importance of the $R^{2}$ results was stressed and some instances of poor fit were highlighted. One potential explanation for the observed occurrences of poor fit is the precision limits of the power quality measurement equipment, which is specified with measurement resolutions of $\pm 0.1 \%$ and $\pm 0.01^{\circ}$ for harmonic currents magnitudes and angles, respectively. Given that the tested devices are very low powered in nature, it is reasonable to surmise that such limitations may be exercised in some instances. Moreover, those sets of raw signal measurements, for which a small standard deviation is observed, are also rendered more susceptible to invoking a poor fit within the corre- 
sponding ZIP load models. This hypothesis is supported upon considering how all of the $R^{2}$ values obtained for the tested CFLs, across Tables 3 to 9 , are greater than or equal to 0.97 . This contrasts to the poorer fits observed among some of the LED lamps which, as Table 3 reports, have much lower power ratings than their CFL counterparts.

Another potential source of poor fit relates to the size of the phase angles measured for each harmonic current order, $\phi_{h}$. The error inherent in the raw measured current data propagates to the associated $\widehat{a_{h}}$ and $\widehat{b_{h}}$ components via calculation. The resultant error is more pronounced (in relative terms) among $\widehat{a_{h}}$ terms for instances in which measurements of $\phi_{h}$ tend towards $90^{\circ}$, at which point large swings in $\cos \left(\phi_{h}\right)$ are effected by only small changes in $\phi_{h}$. The same problem is apparent among $\widehat{b_{h}}$ terms, and thereby derived calculations of $\sin \left(\phi_{h}\right)$, for $\phi_{h} \simeq 0^{\circ}$. This issue is exacerbated when taken in combination with a small relative current magnitude, $\left|I_{h}\right|$, within which a comparatively high relative error will be present due to the precision limitations of the testing equipment.

Analysis of the models depicted in Figs. 6, 7 and 8 helps to yields a better sense of the problem. In Fig. 6(b), a relatively poor fit is observed for the $\widehat{b_{5}}$ component $\left(R^{2}=\right.$ 0.45), which arises from the small amount of variability present within the raw dataset. In terms of relative error, the condition is exacerbated by the precision limitations of the measurement equipment, such that the raw data points appear to be widely dispersed within the scatter plot representation. By contrast, an excellent fit $\left(R^{2}=\right.$ 0.97) is depicted for the ZIP model trend of $\widehat{a_{5}}$ in Fig. 6(a), in which a higher degree of variability is present. Upon recovery of the harmonic magnitude and phase angle values in panels (c) and (d) of Fig. 6, a highly accurate fit $\left(R^{2}=0.97\right)$ is obtained for $\left|I_{5}\right|$, while a low $R^{2}$ value of 0.36 is observed for $\phi_{5}$. However, on inspection of the raw data points in Fig. 6(d), a very low degree of variation is witnessed, and the corresponding ZIP model is actually shown to offer a very accurate approximation. The low $R^{2}$ value 
offers a misleading interpretation of the model, as it arises from the very low standard deviation of the raw measured data set.

A similar prognosis is evident for LED lamp 07, where a poor fit $\left(R^{2}=0.25\right)$ is observed for the $\widehat{a_{h}}$ component in Fig. $7(\mathrm{a})$, with a highly accurate representation $\left(R^{2}\right.$ $=0.99$ ) being possible for $\widehat{b_{h}}$ in Fig. $7(\mathrm{~b})$. Once again, a highly accurate model for $\left|I_{3}\right|$ is evident in Fig. 7(c), with a poorer fit $\left(R^{2}=0.60\right)$ obtained for the very small voltage induced fluctuations of $\phi_{3}$ in Fig. $7(\mathrm{~d})$.

Similarly, it is important to note that for some cases, the inadequacies of using ZIP model theory to fit against measurement discontinuities and/or piecewise behavior becomes apparent. One such example, is contained within the ZIP models for harmonic current $I_{9}$, pertaining to LED lamp 07 (Fig. 8), in which a significant discontinuity is present in the measured data set, around nominal voltage. Limitations of this nature, for the ZIP modelling approach are acknowledged in literature [20, 33], but may be treated as an accepted weakness of a quadratic formula based approach. While higher order, polynomial models may offer better resolution, additional coefficients would need to be defined and natural orientation of the ZIP based approach towards an amalgam of constant impedance, current and power behaviors would be lost.

\section{Conclusions and Further Work}

This paper has advanced a verifiable method for characterizing the active power, reactive power and harmonic current distortion attributes of a selection of modern lighting types. Results are presented in the form of ZIP coefficients in Tables 3 to 9 . These are translated into sets of characteristic curves that are plotted against applied voltage level in Figs. 3, 4 and 12, in which they may also be visually compared against the corresponding experimental measurements.

The generally poor harmonic performance of modern lamps may be thought to 
detract from the otherwise excellent low power benefits imparted to customers. As the adoption of such technologies proliferates, utilities may begin to observe higher technical loss percentages within LV networks [9]. This will manifest as an increased operational cost that is not easily recoverable under present electricity market paradigms. If the findings of this research can be extrapolated to the distribution network level, it may be argued that the lowering of service level voltages, through innovations such as CVR, can assist in mitigating some of the future increases in harmonic distortion. However, one should invoke caution regarding the applicability of this research in relation to LV networks within which very high levels of $T H D_{V}$ are present. As stated in \$2.3, a comprehensive analysis can only be performed for circumstances in which a considerably more complex model for harmonic current phasor characterization is developed.

Given their apparent technical superiority; alongside other factors such as longer lifetimes and waste disposal concerns related to CFLs, one can anticipate how LED technology is likely to supplant CFLs in popularity as manufacturing costs decline. As such, it is the characteristics of the LED lamps presented in this paper that ought to be most seriously considered in the context of future network load growth. As residential uptake of LED lamps grows, load models used by utilities may require adjustment to encompass larger quotients of constant power type behavior. The emergence of LED street lighting will further accentuate this transition. Consequentially, the continuing effectiveness of network management applications, such as CVR, in indirectly reducing customer demand might be expected to decline.

Despite recent efforts to address the historically lax harmonic current restrictions for low powered luminaires, the new limits specified in [16] remain more lenient than those defined for devices with power ratings greater than $25 \mathrm{~W}$. This is amidst projections that LED types will become the dominant lighting technology over the next twenty years [14]. Although such advancements are associated with higher device efficiencies, 
one should not discount the potential applicability of Jevon's Paradox to this discussion [34]. As such, issues pertaining to higher distortion levels within LV networks may be set to emerge as customers migrate from GIL and CFL types. However, ample evidence is presented, in charts such as Figs. 9 and 10 , to suggest that the greater propensity for harmonic diversity among LED lamps, rather than CFLs, should help to assuage some of these concerns about large increases in harmonic current flows within LV networks.

Several potential areas of further work emanate from the analysis presented in this paper. The developed models can be used to more comprehensively define the characteristics of connected load instances within distribution network models, in preparation for analysis with power systems load flow softwares. The characterization results, presented in Tables 3 to 9, serve as reference data in this regard. For large network models, the use of harmonic diversity factors, alongside experimental power quality data accrued for different types of consumer appliance, can be used to produce realistic, aggregate lump load instances, within which the demand attributes of a whole LV feeder (for example) may be encapsulated, as a means of reducing the complexity of the overall network model. Finally, as discussed in $\$ 2.3$, the development of a more extensive modeling framework, appropriate for the resolution of harmonic currents under high background levels of $T H D_{V}$, warrants investigation but is likely to encounter computational challenges that may detract from its intended convenience.

\section{Acknowledgement}

This paper was compiled as part of research within Ofgem's Low Carbon Network Fund Tier 2 project, "Smart Street". The authors gratefully acknowledge the contributions of their project partners at Electricity North West Ltd. and the University of Manchester. Instrumentation used in this project was supported via the GCHQ Equip-

ment Fund. Additional support was provided via EPSRC Suppleheat (EP/M000656/1), 
CAPRICA (EP/M002837/1) and CASE DINOSAUR programs. G. McLorn thanks the

Department of the Economy (Northern Ireland) for supporting his research.

\section{References}

[1] A. Bokhari, et al., Experimental determination of the ZIP coefficients for modern residential, commercial, and industrial loads, IEEE Trans. Power Del. 29 (3) (2014) 1372-1381.

[2] F. L. Quilumba, W.-J. Lee, J. Jativa-Ibarra, Load models for flat-panel TVs, IEEE Trans. Ind. Appl. 50 (6) (2014) 4171-4178.

[3] Y. Wang, J. M. Alonso, X. Ruan, A review of led drivers and related technologies, IEEE Trans. Ind. Electron. 64 (7) (2017) 5754-5765.

[4] S. Uddin, H. Shareef, A. Mohamed, Power quality performance of energy-efficient low-wattage LED lamps, J. Meas. 46 (10) (2013) 3783-3795.

[5] A. Collin, C. Cresswell, S. Djokic, Harmonic cancellation of modern switch-mode power supply load, in: Proc. $14^{\text {th }}$ Int. Conf. Harmonics and Quality of Power, Bergamo, 2010, pp. 94-102.

[6] A. Collin, S. Djokic, Cancellation and attenuation of harmonics in low voltage networks, in: Proc. $16^{\text {th }}$ Int. Conf. Harmonics and Quality of Power, Buchar., 2014, pp. 137-141.

[7] M. Schinkelshoek, N. Watson, B. Heffernan, The characteristics of CFLs; beyond the harmonics, in: Proc. $20^{t h}$ Australas. Univ. Power Eng. Conf. (AUPEC), Christch., N.Z., 2010.

[8] J. Molina, L. Sainz, Compact fluorescent lamp modeling for large scale harmonic penetration studies, IEEE Trans. Power Del. 30 (3) (2015) 1523-1531.

[9] N. R. Watson, T. L. Scott, S. J. Hirsch, Implications for distribution networks of high penetration of Compact Fluorescent Lamps, IEEE Trans. Power Del. 24 (3) (2009) 1521-1528.

[10] J. Meyer, P. Schegner, K. Heidenreich, Harmonic summation effects of modern lamp technologies and small electronic household equipment, in: Proc. $21^{\text {st }}$ Int. Conf. Electr. Distrib. (CIRED), Frankf., Ger., 2011.

[11] V. Cuk, et al., An analysis of diversity factors applied to harmonic emission limits for energy saving lamps, in: Proc. 14 ${ }^{\text {th }}$ Int. Conf. on Harmonics and Quality of Power, Bergamo, 2010.

[12] J. Hunt, Efficient lighting - the way ahead, IET Wiring Matters (46) (2013) 12-14.

[13] M. Yamada, K. Stober, Adoption of Light-Emitting Diodes in Common Lighting Applications, Tech. rep., U.S. Dept. of Energy (Jul. 2015).

[14] J. Penning, K. Stober, V. Taylor, M. Yamada, Energy Savings Forecast of Solid-State Lighting in General Illumination Applications, Tech. rep., U.S. Dept. of Energy (Sep. 2016).

[15] G. McLorn, et al., Enhanced ZIP load modelling for the analysis of harmonic distortion under Conservation Voltage Reduction, CIRED - Open Access Proc. J. 2017 (1) (2017) 1094-1097.

[16] Int. Electrotech. Comm. (IEC), Electromagnetic compatibility (EMC) - Part 3-2: Limits - Limits for harmonic current emissions (equipment input current $\leq 16 \mathrm{~A}$ per phase) ed. 5.0, Int. Stand. IEC 61000-3-2:2018 (2018).

[17] Int. Electrotech. Comm. (IEC) Tech Rep., Electromagnetic compatibility (EMC) - Part 3-15: Limits - Assessment of low frequency electromagnetic immunity and emission requirements for dispersed generation systems in LV networks, IEC/TR 61000-3-15:2011 (2011). 
[18] IEEE Power \& Energy Soc., IEEE Standard Definitions for the Measurement of Electric Power Quantities Under Sinusoidal, Nonsinusoidal, Balanced, or Unbalanced Conditions, IEEE Stand. 1459-2010, IEEE (2010).

[19] L. Waters, Energy consumption in the UK, Tech. rep., U.K. Dept. for Business, Energy and Industrial Strategy (BEIS) (Nov. 2016).

[20] I. Task Force on Load Representation for Dynamic Performance, Standard load models for power flow and dynamic performance simulation, IEEE Trans. Power Syst. 10 (3) (1995) 1302-1313.

[21] I. Task Force on Load Representation for Dynamic Performance, Load representation for dynamic performance analysis, IEEE Trans. Power Syst. 8 (2) (1993) 472-482.

[22] Int. Electrotech. Comm. (IEC), Electromagnetic compatibility (EMC) - Part 3-2: Limits - Limits for harmonic current emissions (equipment input current $\leq 16$ A per phase) ed. 4.0, Int. Stand. IEC 61000-3-2:2014 (2014).

[23] Eur. Comm. for Electrotech. Stand. (CENELEC), Voltage characteristics of electricity supplied by public electricity networks, Eur. Stand. (EN) 50160:2010+A1 (2015).

[24] M. Brunoro, L. F. Encarnacao, J. F. Fardin, Modeling of loads dependent on harmonic voltages, J. Electr. Power Syst. Res. 152 (11) (2017) 367-376.

[25] A. M. Blanco, J. Meyer, P. Schegner, Aggregation characteristic of low-order harmonic currents in residential low-voltage networks, in: Proc. IEEE Power and Energy Soc. (PES) PowerTech Conf., Manch., U.K., 2017.

[26] M. T. Au, J. V. Milanovic, Establishing harmonic distortion level of distribution network based on stochastic aggregate harmonic load models, IEEE Trans. Power Del. 22 (2) (2007) 1086-1092.

[27] D. Salles, C. Jiang, W. Xu, W. Freitas, H. E. Mazin, Assessing the collective harmonic impact of modern residential loads - Part I: Methodology, IEEE Trans. Power Del. 27 (4) (2012) 1937-1946.

[28] A. Collin, I. Hernando-Gil, J. Acosta, S. Djokic, An $11 \mathrm{kV}$ steady state residential aggregate load model. Part 1: Aggregation methodology, in: Proc. IEEE Power and Energy Soc. (PES) PowerTech Conf., Trondheim, 2011.

[29] A. M. Blanco, et al., Stochastic harmonic load model of residential users based on measurements, in: Proc. IEEE Power and Energy Soc. (PES) PowerTech Conf., Eindhoven, 2015.

[30] IES Testing Procedures Committee, Approved method: Electrical and photometric measurements of solid-state lighting products, IES LM-79-08, Illuminating Eng. Soc. (IES) (2007).

[31] Semicond. Equip. \& Mater. Int. (SEMI), Specification for semiconductor processing equipment voltage sag immunity, SEMI Stand. F47, SEMI (2000).

[32] F. C. De La Rosa, Harmonics and Power Systems, CRC Press, 2006, pp. 22-37.

[33] G. McLorn, S. McLoone, Small-signal refinement of power system static load modelling techniques, in: Advanced Computational Methods in Energy, Power, Electric Vehicles, and Their Integration, Springer, 2017, pp. 467-476.

[34] Keeping the lights on - an inconvenient truth, remarks by N. Burton at the IET President's Address 2010 lecture, London (Oct. 2010). 


\section{Figures and Tables}

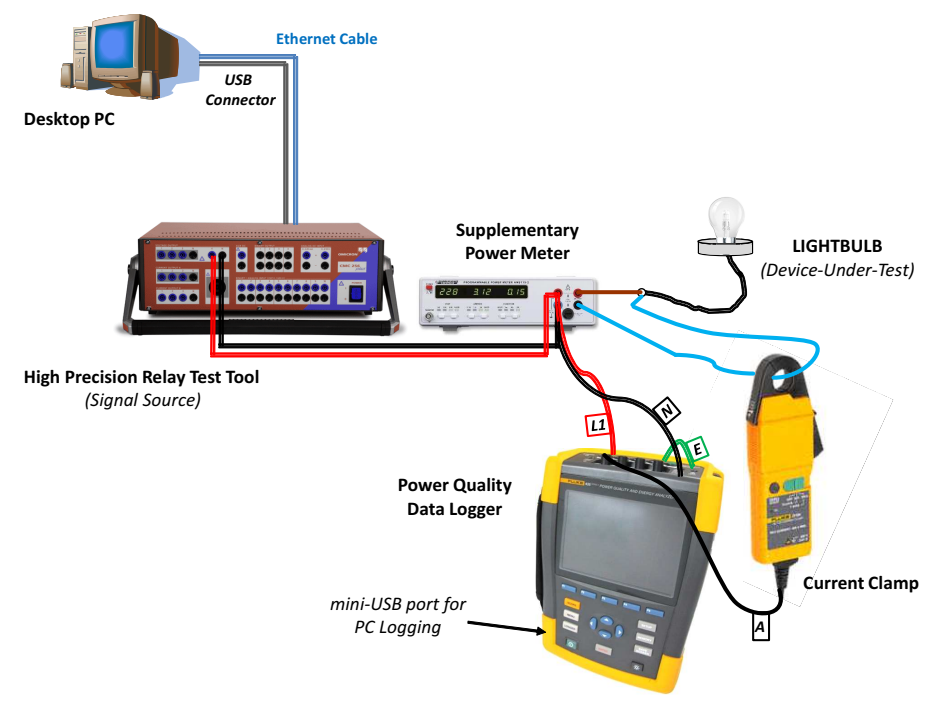

Figure 1: Experimental Apparatus - Connectivity Diagram

Table 1: Harmonic Current Limits for IEC 61000-3-2/Class C devices (> $25 \mathrm{~W}$ )

(\% of Fundamental Current, $\mathrm{I}_{1}$ )

\begin{tabular}{|c|c|}
\hline $\begin{array}{c}\text { Harmonic Order } \\
\boldsymbol{n}\end{array}$ & $\begin{array}{c}\text { Max. Harmonic } \\
\text { Current }\left(\% \text { of } \mathrm{I}_{1} \text { ) }\right.\end{array}$ \\
\hline 2 & 2 \\
3 & $30 \cdot \lambda$ \\
5 & 10 \\
7 & 7 \\
9 & 5 \\
$11 \leq \mathrm{n}($ odd $) \leq 39$ & 3 \\
\hline
\end{tabular}

Table 2: Inferred Harmonic Current Limits for IEC 61000-3-2/Class C devices ( $\geq 5 \mathrm{~W}$ and $\leq 25 \mathrm{~W}$ ); Nominal Voltage $=230 \mathrm{~V}\left(\%\right.$ of Fundamental Current, $\left.\mathrm{I}_{1}\right)$

\begin{tabular}{|c|ccc|}
\hline Harmonic Order & \multicolumn{3}{|c|}{ Max. Harmonic Current (\% of $\left.\mathrm{I}_{1}\right)$} \\
$\boldsymbol{n}$ & $(\boldsymbol{i})$ & (ii) & (iii) \\
\hline 3 & 78.2 & 86.0 & 35.0 \\
5 & 43.7 & 61.0 & 25.0 \\
7 & 23.0 & & 30.0 \\
9 & 11.5 & & 20.0 \\
11 & 8.1 & & 20.0 \\
$13 \leq \mathrm{n}$ (odd) $\leq 39$ & $88.6 / n$ & & \\
\hline
\end{tabular}




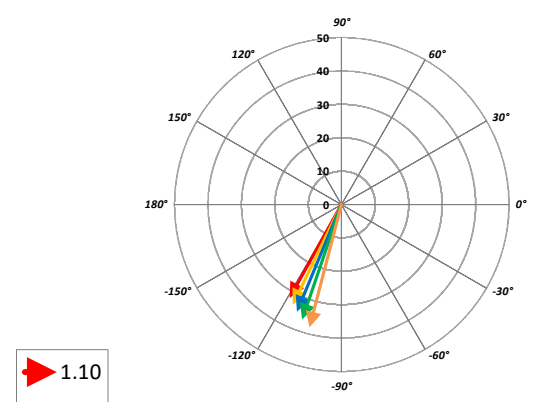

(a)

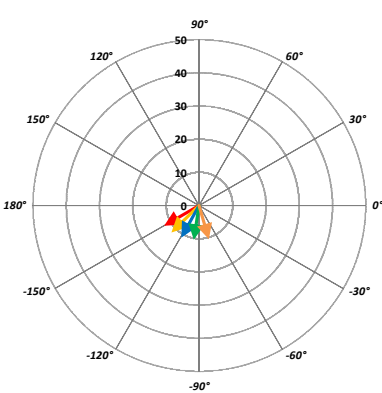

(d)

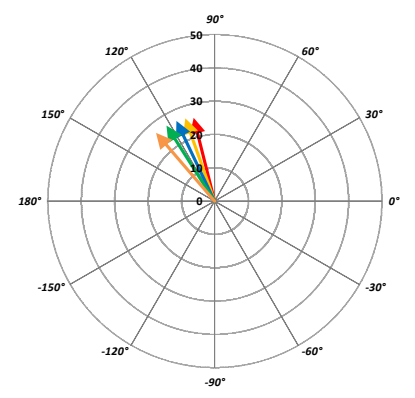

(b)

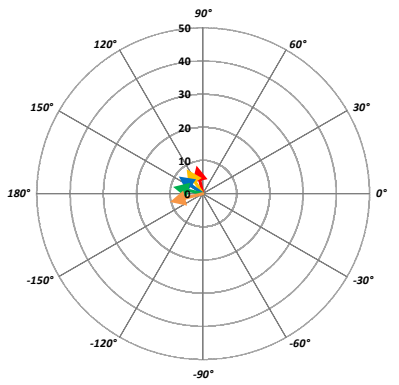

(e)

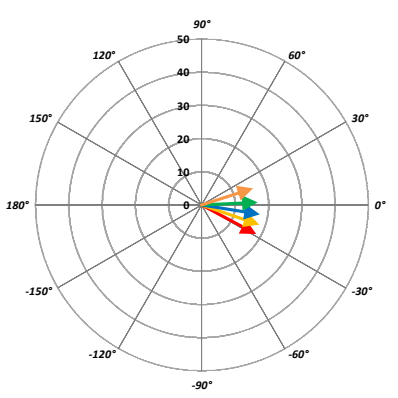

(c)

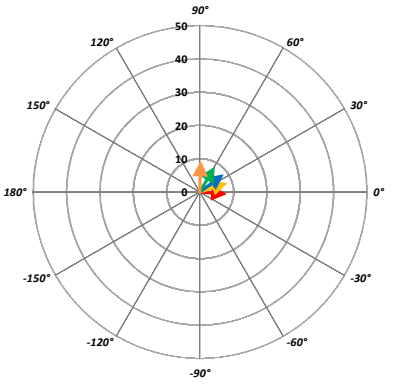

(f)

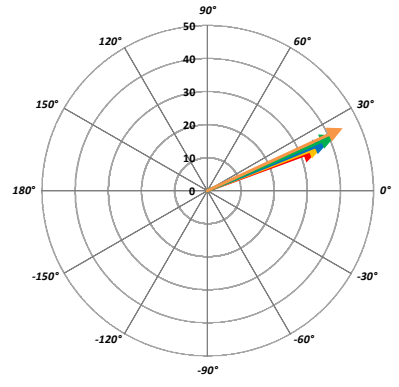

(g)

Figure 2: Bulb 05. Harmonic currents in polar form (magnitude in $\mathrm{mA}$, phase angle in degrees, reference current of $50 \mathrm{~mA}$ ). Orders (a) 3, (b) 5, (c) 7, (d) 9, (e) 11, (f) 13 and (g) fundamental. Observed under application of voltages between 0.90 and $1.10 \mathrm{pu}$.

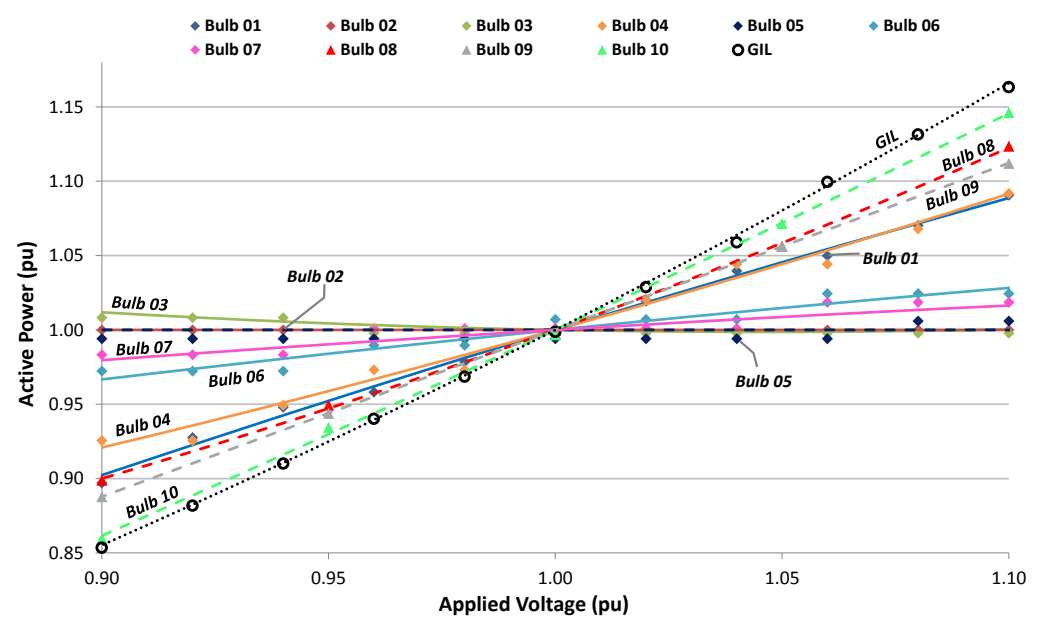

Figure 3: Per-unit active power vs. voltage; LED, CFL and GIL lamps 


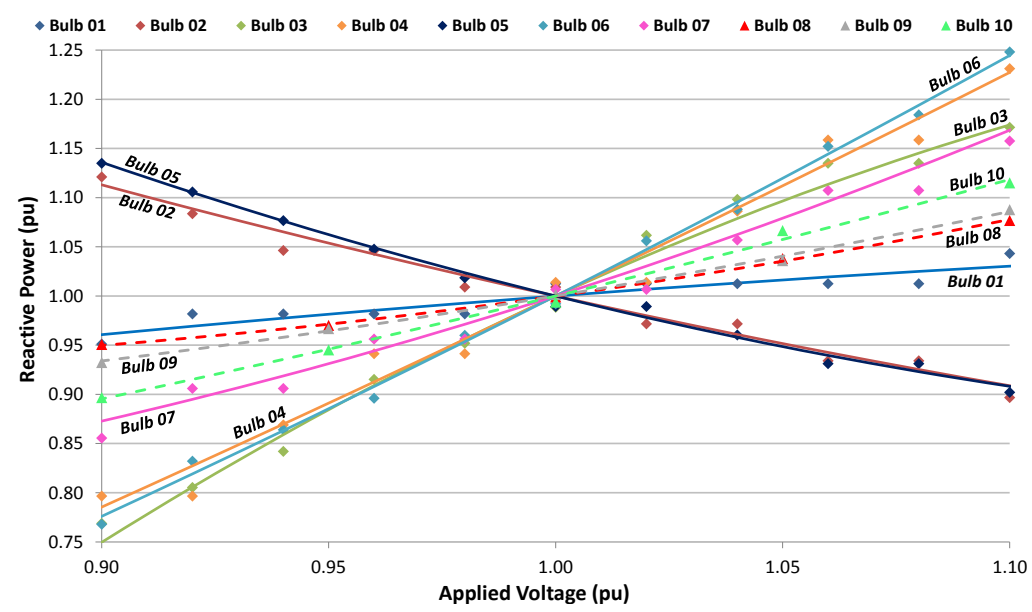

Figure 4: Per-unit reactive power vs. voltage; LED and CFL lamps

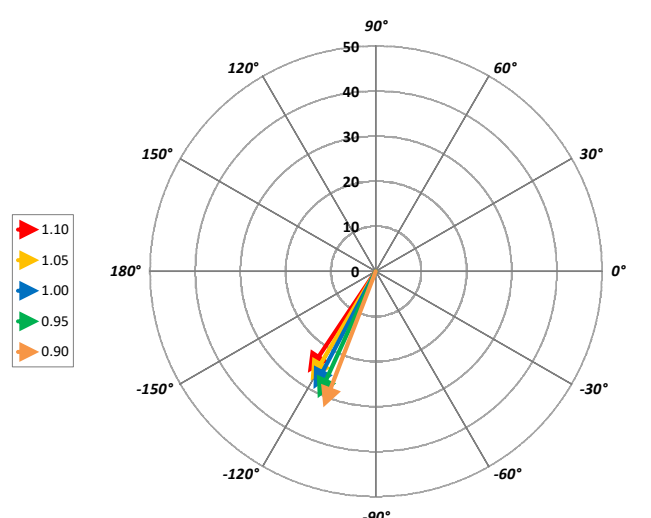

(a)

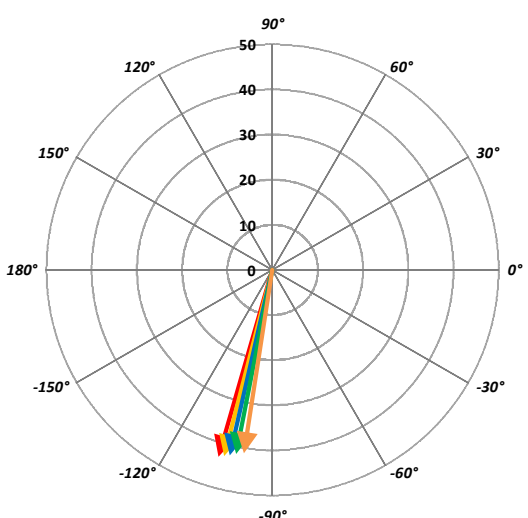

(b)

Figure 5: $3^{\text {rd }}$ order harmonic currents (magnitude in $\mathrm{mA}$, phase angle in degrees). Observed under application of voltages between 0.90 and $1.10 \mathrm{pu}$ for (a) LED lamp 02 and (b) CFL lamp 09.

Table 3: ZIP models for Active and Reactive Power Consumption

\begin{tabular}{|c|l|c|c|c|cccc|c|c|ccc|}
\hline Bulb & Bulb Type & Rating (W) & $\mathbf{P}_{0}$ & $\mathbf{R}_{p}^{2}$ & $\mathbf{Z}_{p}$ & $\mathbf{I}_{p}$ & $\mathbf{P}_{p}$ & $\mathbf{Q}_{0}$ & $\mathbf{R}_{q}^{2}$ & $\mathbf{Z}_{q}$ & $\mathbf{I}_{q}$ & $\mathbf{P}_{q}$ \\
\hline 01 & LED/Globe & 9.0 & 9.81 & 1.00 & -0.446 & 1.823 & -0.377 & -3.26 & 0.85 & -0.447 & 1.242 & 0.205 \\
02 & LED/Globe & 8.0 & 7.10 & 1.00 & 0.000 & 0.000 & 1.000 & -2.67 & 0.98 & 1.091 & -3.204 & 3.113 \\
03 & LED/Globe & 10.0 & 9.50 & 0.81 & 0.613 & -1.284 & 1.671 & -2.74 & 0.99 & -3.826 & 9.773 & -4.947 \\
04 & LED/Downlight & 5.5 & 4.21 & 0.99 & 0.623 & -0.393 & 0.770 & -1.38 & 0.98 & 0.634 & 0.941 & -0.575 \\
05 & LED/Globe & 8.5 & 8.45 & 1.00 & 0.000 & 0.000 & 1.000 & -3.43 & 0.99 & 2.207 & -5.552 & 4.345 \\
06 & LED/Globe & 5.7 & 5.76 & 0.94 & -0.253 & 0.814 & 0.439 & -3.12 & 1.00 & 1.026 & 0.291 & -0.317 \\
07 & LED/Downlight & 6.0 & 5.70 & 0.87 & -0.195 & 0.574 & 0.621 & -1.98 & 0.98 & 2.068 & -2.658 & 1.589 \\
08 & CFL/Spiral & 20.0 & 17.80 & 1.00 & 1.124 & -1.135 & 1.011 & -10.31 & 1.00 & 1.386 & -2.131 & 1.746 \\
09 & CFL/Triple Tube & 11.0 & 10.70 & 1.00 & 0.000 & 1.121 & -0.121 & -5.79 & 1.00 & 0.987 & -1.214 & 1.227 \\
10 & CFL/Globe & 9.0 & 8.03 & 1.00 & 0.356 & 0.708 & -0.064 & -4.13 & 1.00 & 0.693 & -0.270 & 0.578 \\
11 & GIL & 60.0 & 56.41 & 1.00 & 1.064 & -0.575 & 0.511 & -0.34 & 0.85 & 4.290 & -5.233 & 1.944 \\
\hline
\end{tabular}




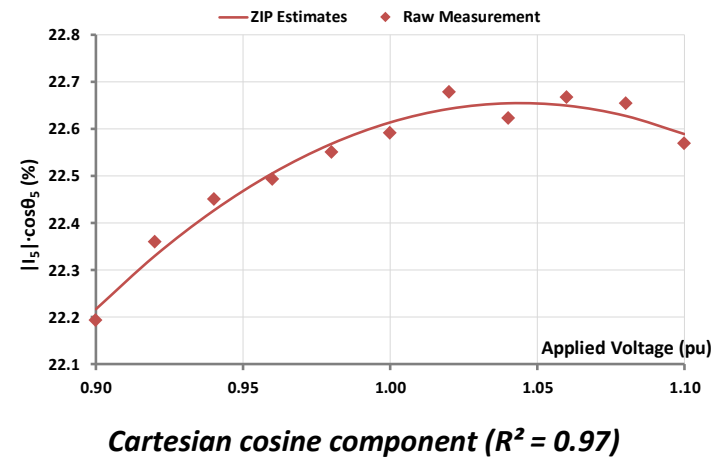

(a)

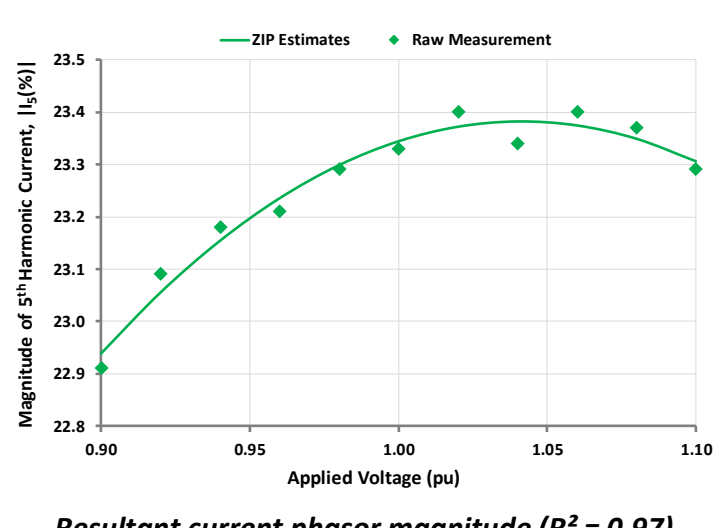

Resultant current phasor magnitude $\left(R^{2}=0.97\right)$

(c)

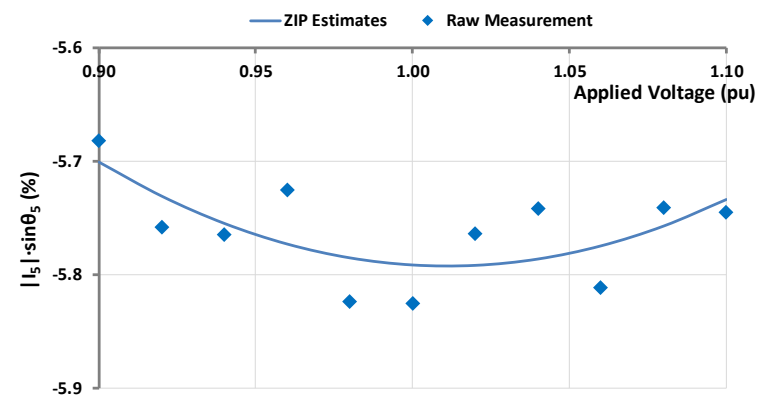

Cartesian sine component $\left(R^{2}=0.45\right)$

(b)

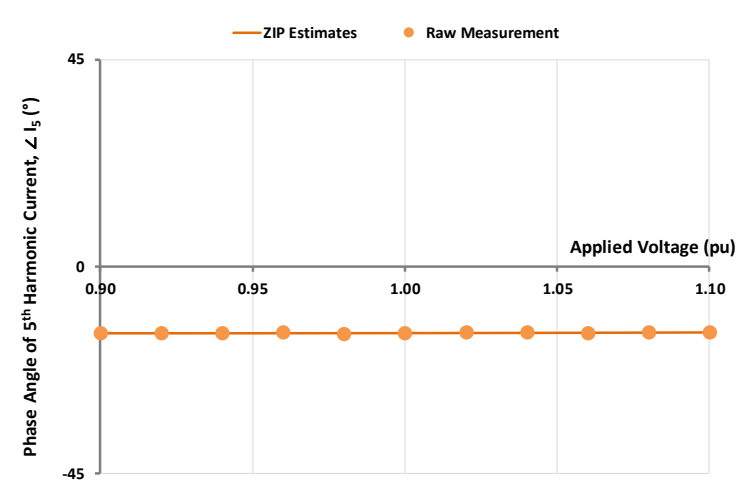

Resultant current phasor angle $\left(R^{2}=0.36\right)$

(d)

Figure 6: LED lamp 04. Measured values and ZIP fitted trends for $5^{\text {th }}$ harmonic current vs. voltage: (a) $\left|\widehat{I_{5}}\right| \cdot \cos \widehat{\phi_{5}}$ component, (b) $\left|\widehat{I_{5}}\right| \cdot \sin \widehat{\phi_{5}}$ component, (c) Phasor magnitude, $\left|\widehat{I_{5}}\right|(\%)$, and (d) Phasor phase angle, $\angle \widehat{\phi_{5}}\left({ }^{\circ}\right)$.

Table 4: ZIP model representations of $3^{\text {rd }}$ order harmonic current, as $\%$ of Fundamental

\begin{tabular}{|c|l|c|c|c|ccc|c|c|ccc|c|}
\cline { 2 - 11 } \multicolumn{2}{c|}{} & \multicolumn{9}{c|}{$\widehat{a_{h}} \equiv\left|\widehat{I_{h}}\right| \cdot \cos \widehat{\phi_{h}}$} & \multicolumn{5}{c|}{$\widehat{b_{h}} \equiv\left|\widehat{I_{h}}\right| \cdot \sin \widehat{\phi_{h}}$} \\
\hline Bulb & Bulb Type & $\mathbf{a}_{0, h=3}(\%)$ & $\mathbf{R}_{a, 3}^{2}$ & $\mathbf{Z}_{a, 3}$ & $\mathbf{I}_{a, 3}$ & $\mathbf{P}_{a, 3}$ & $\mathbf{b}_{0, h=3}(\%)$ & $\mathbf{R}_{b, 3}^{2}$ & $\mathbf{Z}_{b, 3}$ & $\mathbf{I}_{b, 3}$ & $\mathbf{P}_{b, 3}$ & $\left|\mathbf{I}_{0,3}\right|(\%)$ & $\left.\angle \mathbf{I}_{0,3}{ }^{\circ}\right)$ \\
\hline 01 & LED/Globe & -51.1 & 1.00 & -0.358 & 1.668 & -0.310 & -74.6 & 1.00 & -0.200 & 0.136 & 1.065 & 90.4 & -124.4 \\
02 & LED/Globe & -41.8 & 1.00 & -2.645 & 7.609 & -3.963 & -79.1 & 1.00 & -0.504 & 0.672 & 0.832 & 89.5 & -117.8 \\
03 & LED/Globe & 7.8 & 0.99 & 1.866 & -5.074 & 4.207 & -1.4 & 0.97 & 87.300 & -167.46 & 81.163 & 8.0 & -10.3 \\
04 & LED/Downlight & 37.1 & 0.97 & -0.226 & 0.209 & 1.017 & -5.6 & 0.60 & -3.284 & 6.220 & -1.936 & 37.5 & -8.5 \\
05 & LED/Globe & -33.7 & 1.00 & -4.149 & 11.548 & -6.398 & -80.9 & 1.00 & -0.758 & 1.311 & 0.447 & 87.7 & -112.6 \\
06 & LED/Globe & 47.9 & 1.00 & -1.688 & 3.607 & -0.919 & -15.2 & 1.00 & -1.248 & 3.050 & -0.802 & 50.3 & -17.6 \\
07 & LED/Downlight & 1.0 & 0.25 & 33.011 & -66.550 & 34.539 & -11.1 & 0.99 & 0.554 & 1.929 & -1.483 & 11.1 & -84.7 \\
08 & CFL/Spiral & -5.0 & 1.00 & -23.039 & 58.043 & -34.004 & -75.5 & 1.00 & -0.780 & 1.806 & -0.027 & 75.7 & -93.8 \\
09 & CFL/Triple Tube & -17.8 & 1.00 & -3.290 & 9.515 & -5.226 & -77.7 & 1.00 & -0.282 & 0.631 & 0.651 & 79.7 & -102.9 \\
10 & CFL/Globe & -9.7 & 1.00 & -4.241 & 11.918 & -6.677 & -79.7 & 1.00 & -0.256 & 0.622 & 0.634 & 80.3 & -97.0 \\
11 & GIL & 0.1 & 0.94 & 0.378 & 0.875 & -0.253 & -1.1 & 0.99 & 0.907 & -0.899 & 0.992 & 1.1 & -83.7 \\
\hline
\end{tabular}




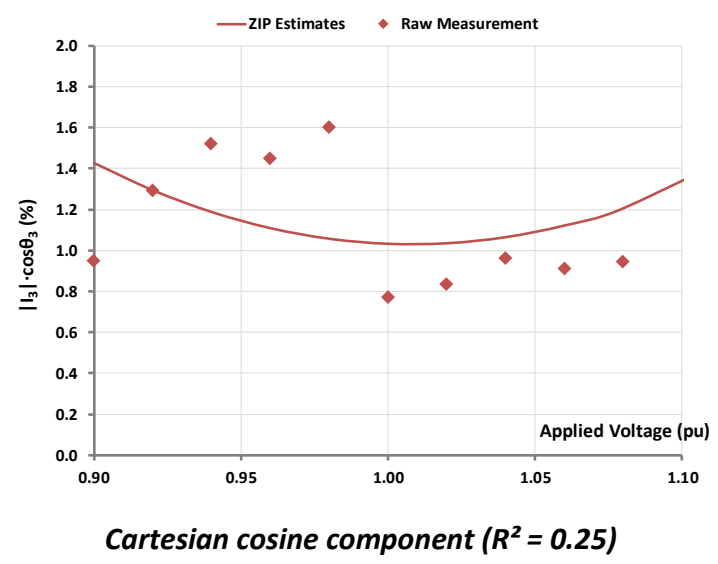

(a)

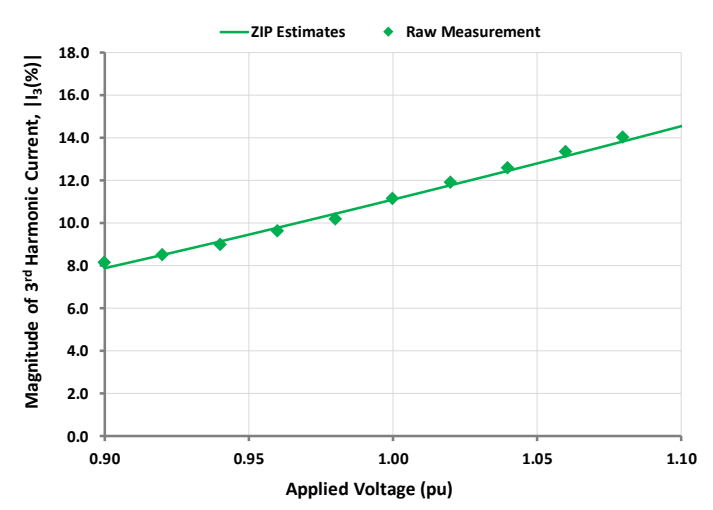

Resultant current phasor magnitude $\left(R^{2}=0.99\right)$

(c)

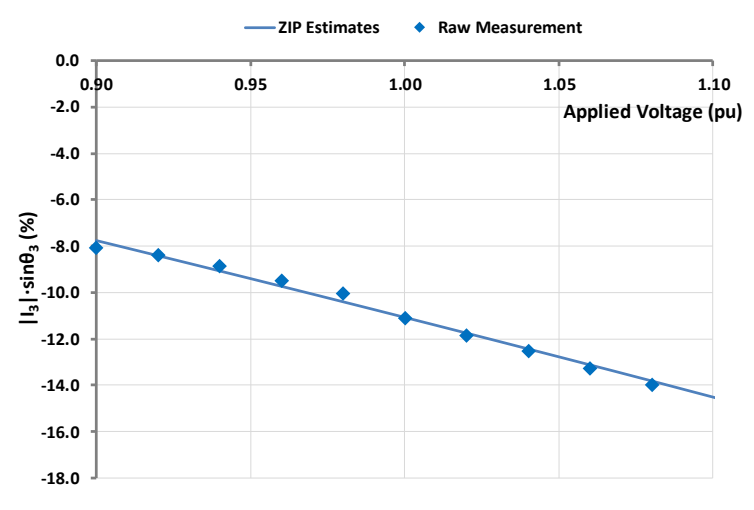

Cartesian sine component $\left(R^{2}=0.99\right)$

(b)

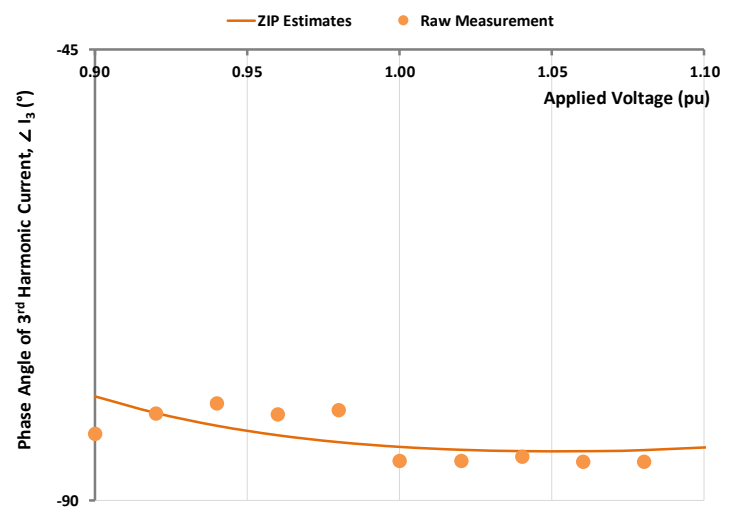

Resultant current phasor angle $\left(R^{2}=0.60\right)$

(d)

Figure 7: LED lamp 07. Measured values and ZIP fitted trends for $3^{\text {rd }}$ harmonic current vs. voltage: (a) $\left|\widehat{I_{3}}\right| \cdot \cos \widehat{\phi_{3}}$ component, (b) $\left|\widehat{I_{3}}\right| \cdot \sin \widehat{\phi_{3}}$ component, (c) Phasor magnitude, $\left|\widehat{I_{3}}\right|(\%)$, and (d) Phasor phase angle, $\angle \widehat{\phi_{3}}\left({ }^{\circ}\right)$.

Table 5: ZIP model representations of $5^{\text {th }}$ order harmonic current, as $\%$ of Fundamental

\begin{tabular}{|c|c|c|c|c|c|c|c|c|c|c|c|c|c|}
\hline \multirow[b]{2}{*}{ Bulb } & \multirow[b]{2}{*}{ Bulb Type } & \multicolumn{5}{|c|}{$\widehat{a_{h}} \equiv\left|\widehat{I_{h}}\right| \cdot \cos \widehat{\phi_{h}}$} & \multicolumn{5}{|c|}{$\widehat{b_{h}} \equiv\left|\widehat{I_{h}}\right| \cdot \sin \widehat{\phi_{h}}$} & \multirow[b]{2}{*}{$\left|\mathbf{I}_{0,5}\right|(\%)$} & \multirow[b]{2}{*}{$\angle \mathbf{I}_{0,5}\left(^{\circ}\right)$} \\
\hline & & $\mathbf{a}_{0, h=5}(\%)$ & $\mathbf{R}_{a, 5}^{2}$ & $\mathbf{Z}_{a, 5}$ & $\mathbf{I}_{a, 5}$ & $\mathbf{P}_{a, 5}$ & $\mathbf{b}_{0, h=5}(\%)$ & $\mathbf{R}_{b, 5}^{2}$ & $\mathbf{Z}_{b, 5}$ & $\mathbf{I}_{b, 5}$ & $\mathbf{P}_{b, 5}$ & & \\
\hline 01 & LED/Globe & -5.9 & 1.00 & -2.059 & -8.221 & 11.279 & 73.7 & 1.00 & -0.790 & 2.046 & -0.256 & 73.9 & 94.5 \\
\hline 02 & LED/Globe & -19.6 & 1.00 & -0.289 & -5.510 & 6.799 & 68.9 & 1.00 & -3.183 & 7.681 & -3.498 & 71.6 & 105.9 \\
\hline 03 & LED/Globe & -0.13 & 0.28 & -223.35 & 438.53 & -214.18 & -0.05 & 0.97 & 2395.4 & -4554.4 & 2160.0 & 0.1 & -160.4 \\
\hline 04 & LED/Downlight & 22.6 & 0.97 & -0.931 & 1.945 & -0.014 & -5.8 & 0.45 & -1.270 & 2.568 & -0.298 & 23.3 & -14.4 \\
\hline 05 & LED/Globe & -29.0 & 1.00 & -1.866 & 0.023 & 2.843 & 60.6 & 1.00 & -4.426 & 10.865 & -5.439 & 67.2 & 115.6 \\
\hline 06 & LED/Globe & 19.9 & 1.00 & -4.276 & 10.856 & -5.580 & -13.1 & 1.00 & -1.649 & 4.366 & -1.717 & 23.8 & -33.3 \\
\hline 07 & LED/Downlight & 1.8 & 0.99 & 8.428 & -13.268 & 5.840 & -8.1 & 0.97 & 0.546 & 0.979 & -0.526 & 8.3 & -77.6 \\
\hline 08 & CFL/Spiral & -39.2 & 0.99 & -1.748 & 3.643 & -0.895 & 18.9 & 1.00 & -7.787 & 20.010 & -11.222 & 43.6 & 154.2 \\
\hline 09 & CFL/Triple Tube & -37.2 & 1.00 & -0.520 & 0.482 & 1.038 & 37.4 & 1.00 & -2.245 & 6.459 & -3.214 & 52.8 & 134.8 \\
\hline 10 & CFL/Globe & -42.0 & 1.00 & -0.500 & 0.940 & 0.560 & 28.4 & 1.00 & -2.180 & 6.115 & -2.934 & 50.7 & 145.9 \\
\hline 11 & GIL & 0.0 & - & 0.000 & 0.000 & 1.000 & 0.0 & - & 0.000 & 0.000 & 1.000 & 0.0 & 45.0 \\
\hline
\end{tabular}




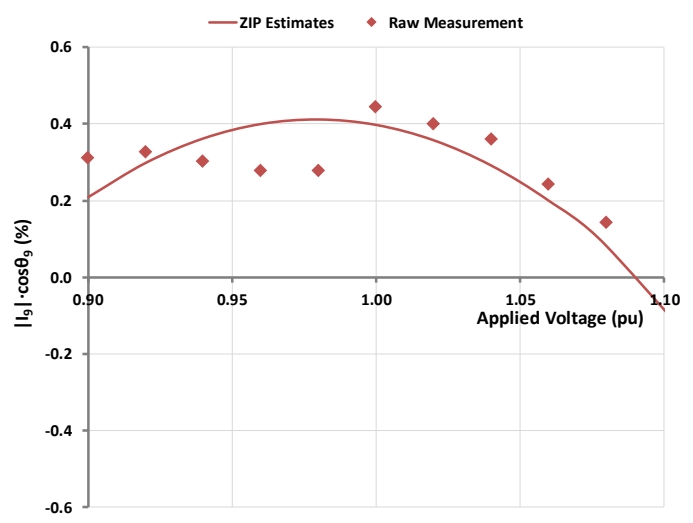

Cartesian cosine component $\left(R^{2}=0.90\right)$

(a)

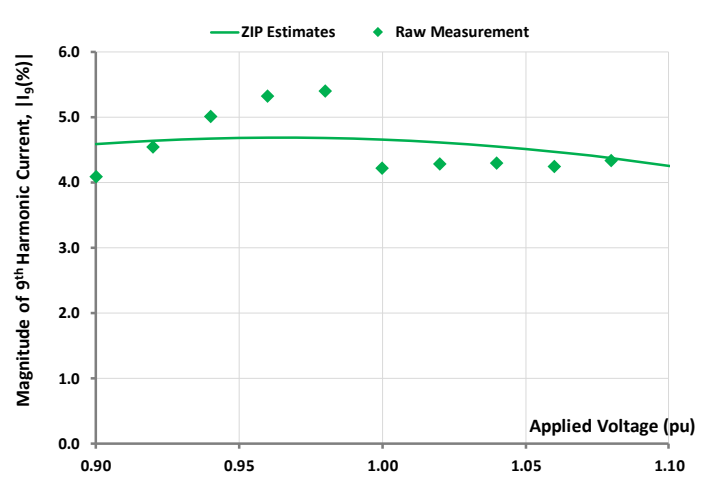

Resultant current phasor magnitude $\left(R^{2}=0.15\right)$

(c)

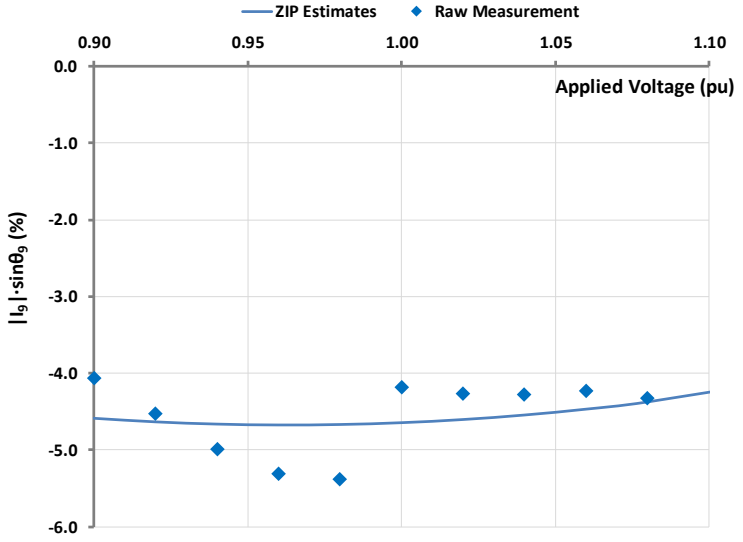

Cartesian sine component $\left(R^{2}=0.18\right)$

(b)

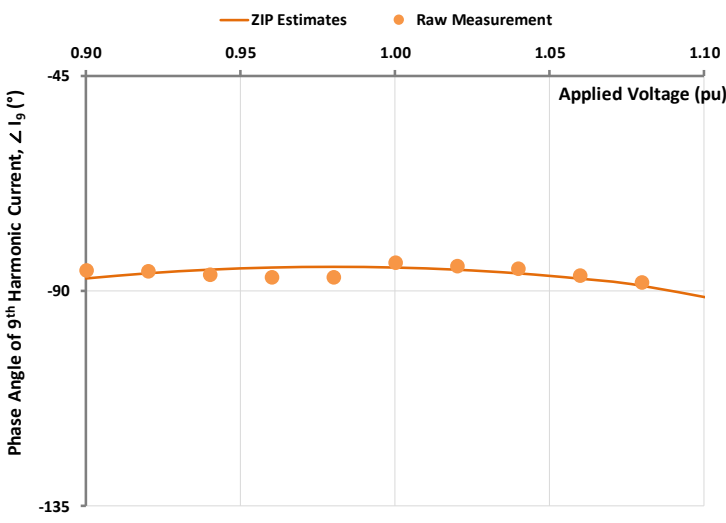

Resultant current phasor angle $\left(R^{2}=0.95\right)$

(d)

Figure 8: LED lamp 07. Measured values and ZIP fitted trends for $9^{\text {th }}$ harmonic current vs. voltage: (a) $\left|\widehat{I_{9}}\right| \cdot \cos \widehat{\phi_{9}}$ component, (b) $\left|\widehat{I_{9}}\right| \cdot \sin \widehat{\phi_{9}}$ component, (c) Phasor magnitude, $\left|\widehat{I_{9}}\right|(\%)$, and (d) Phasor phase angle, $\angle \widehat{\phi_{9}}\left({ }^{\circ}\right)$.

Table 6: ZIP model representations of $7^{\text {th }}$ order harmonic current, as $\%$ of Fundamental

\begin{tabular}{|c|c|c|c|c|c|c|c|c|c|c|c|c|c|}
\hline \multirow[b]{2}{*}{ Bulb } & \multirow[b]{2}{*}{ Bulb Type } & \multicolumn{5}{|c|}{$\widehat{a_{h}} \equiv\left|\widehat{I_{h}}\right| \cdot \cos \widehat{\phi_{h}}$} & \multicolumn{5}{|c|}{$\widehat{b_{h}} \equiv\left|\widehat{I_{h}}\right| \cdot \sin \widehat{\phi_{h}}$} & \multirow[b]{2}{*}{$\left|\mathbf{I}_{0,7}\right|(\%)$} & \multirow[b]{2}{*}{$\angle \mathbf{I}_{0,7}\left({ }^{\circ}\right)$} \\
\hline & & $\mathbf{a}_{0, h=7}(\%)$ & $\mathbf{R}_{a, 7}^{2}$ & $\mathbf{Z}_{a, 7}$ & $\mathbf{I}_{a, 7}$ & $\mathbf{P}_{a, 7}$ & $\mathbf{b}_{0, h=7}(\%)$ & $\mathbf{R}_{b, 7}^{2}$ & $\mathbf{Z}_{b, 7}$ & $\mathbf{I}_{b, 7}$ & $\mathbf{P}_{b, 7}$ & & \\
\hline 01 & LED/Globe & 39.7 & 1.00 & -2.270 & 3.846 & -0.576 & -36.7 & 1.00 & -1.123 & 4.791 & -2.668 & 54.0 & -42.8 \\
\hline 02 & LED/Globe & 45.7 & 0.99 & -6.785 & 13.618 & -5.833 & -21.9 & 1.00 & -5.092 & 18.263 & -12.171 & 50.7 & -25.6 \\
\hline 03 & LED/Globe & 2.2 & 0.99 & -1.105 & 7.568 & -5.463 & 0.4 & 0.94 & -300.06 & 580.23 & -279.17 & 2.2 & 9.4 \\
\hline 04 & LED/Downlight & 14.1 & 1.00 & -1.630 & 3.808 & -1.177 & -5.4 & 0.98 & -2.281 & 5.099 & -1.818 & 15.2 & -21.0 \\
\hline 05 & LED/Globe & 44.1 & 1.00 & -9.039 & 19.354 & -9.315 & -7.2 & 1.00 & -5.163 & 35.271 & -29.107 & 44.7 & -9.2 \\
\hline 06 & LED/Globe & 0.8 & 1.00 & -27.62 & 114.69 & -86.068 & -9.5 & 1.00 & -1.044 & 3.883 & -1.839 & 9.6 & -85.1 \\
\hline 07 & LED/Downlight & 1.3 & 0.52 & 8.973 & -18.955 & 10.981 & -6.6 & 0.94 & -5.095 & 12.572 & -6.477 & 6.7 & -79.1 \\
\hline 08 & CFL/Spiral & 6.7 & 1.00 & -20.338 & 53.013 & -31.675 & 21.2 & 0.98 & -4.395 & 8.506 & -3.110 & 22.2 & 72.5 \\
\hline 09 & CFL/Triple Tube & 25.0 & 1.00 & -3.854 & 10.424 & -5.570 & 14.2 & 1.00 & -1.832 & 0.210 & 2.622 & 28.7 & 29.6 \\
\hline 10 & CFL/Globe & 17.2 & 1.00 & -3.911 & 10.743 & -5.832 & 20.3 & 1.00 & -0.904 & 0.992 & 0.911 & 26.6 & 49.8 \\
\hline 11 & GIL & 0.0 & - & 0.000 & 0.000 & 1.000 & 0.0 & - & 0.000 & 0.000 & 1.000 & 0.0 & 45.0 \\
\hline
\end{tabular}




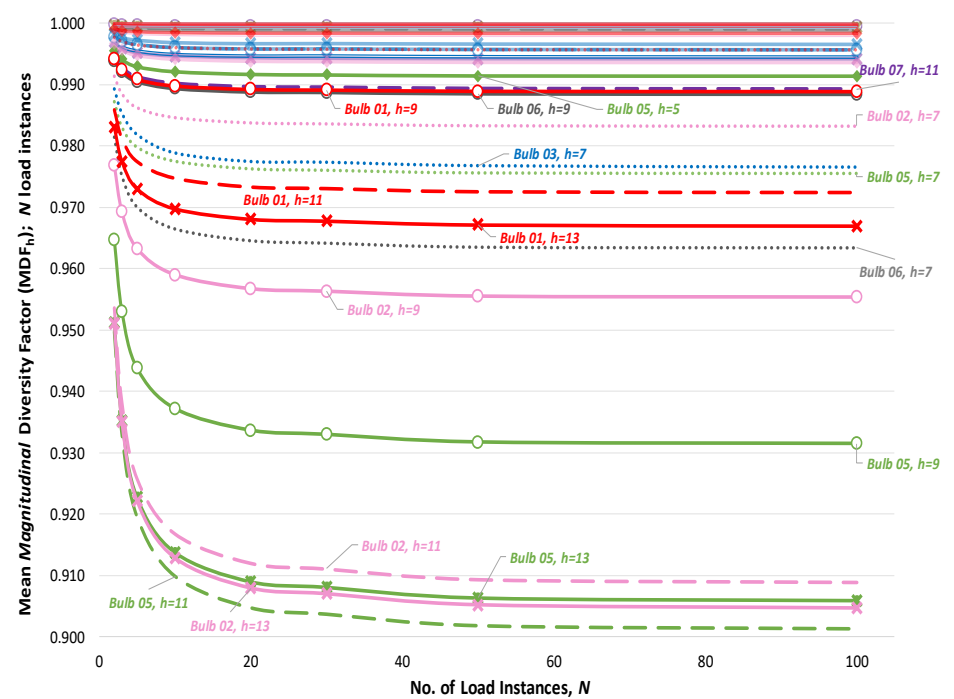

(a)

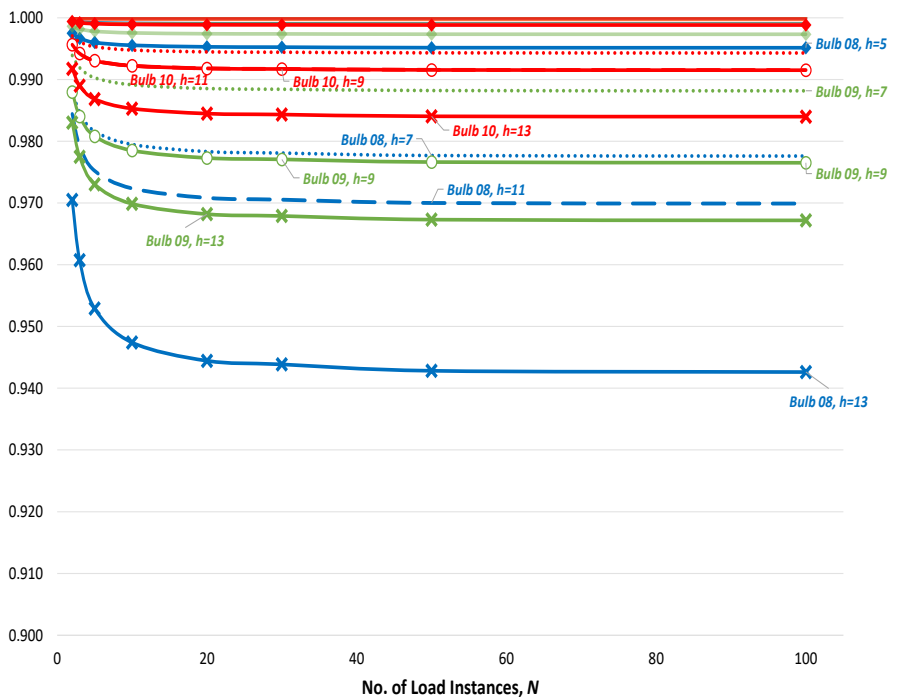

(b)

Figure 9: Mean MDF for harmonic current orders 1, 3, 5, 7, 9, 11 and 13, for number of device instances $N \leq 100$. Homogeneous collections of (a) LED and (b) CFL instances, by lamp type and harmonic current order

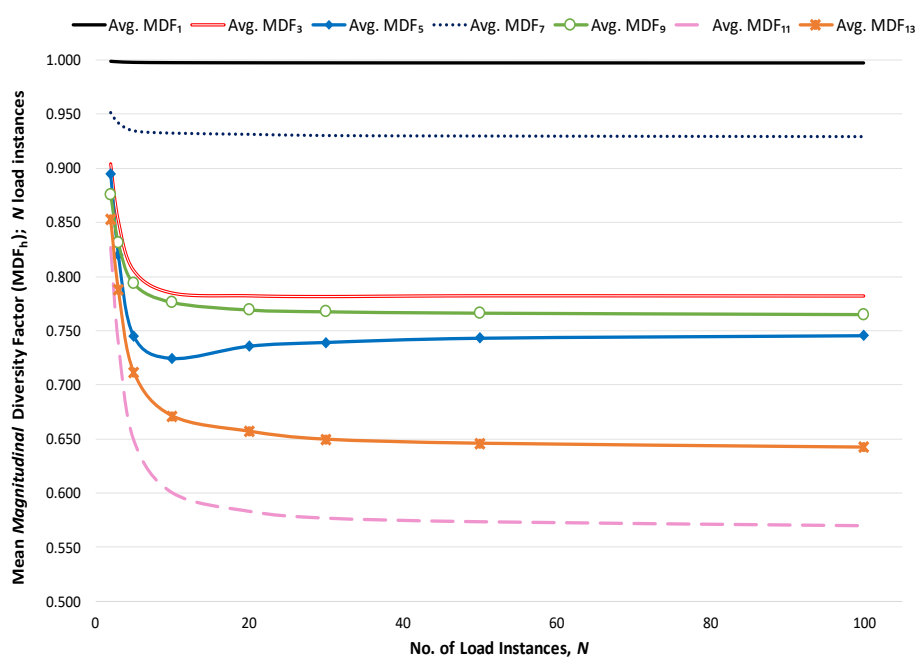

(a)

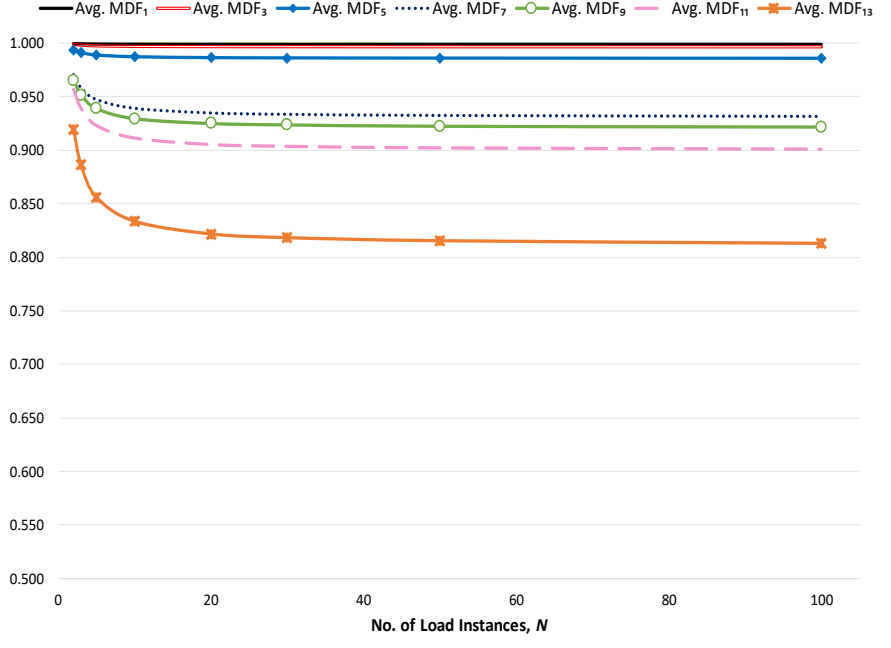

(b)

Figure 10: Mean MDF for harmonic current orders 1, 3, 5, 7, 9, 11 and 13, for number of device instances $N \leq 100$. Mixed lamp type combinations of (a) LED and (b) CFL instances, by harmonic current order 


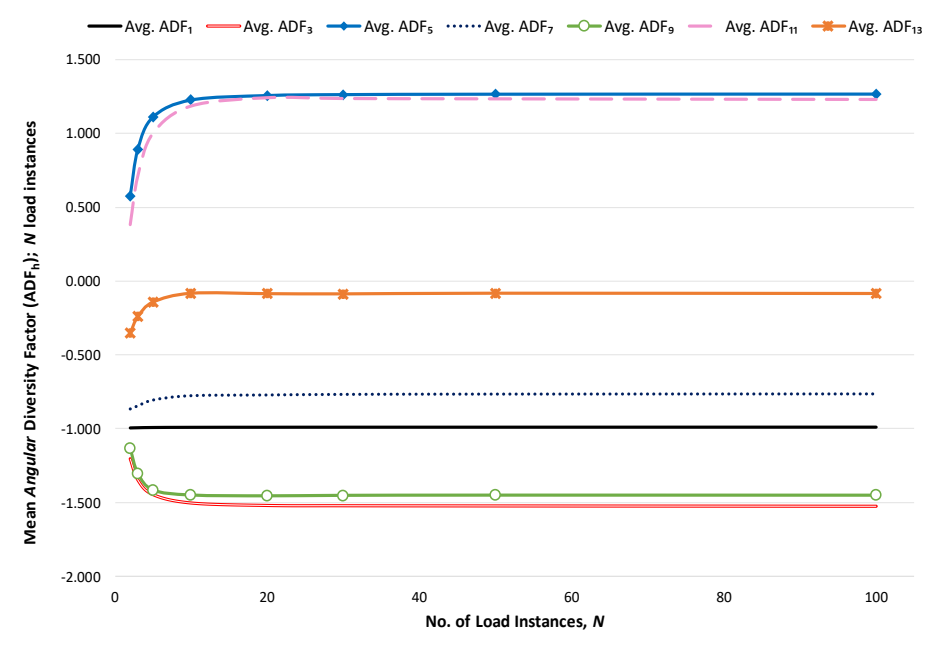

(a)

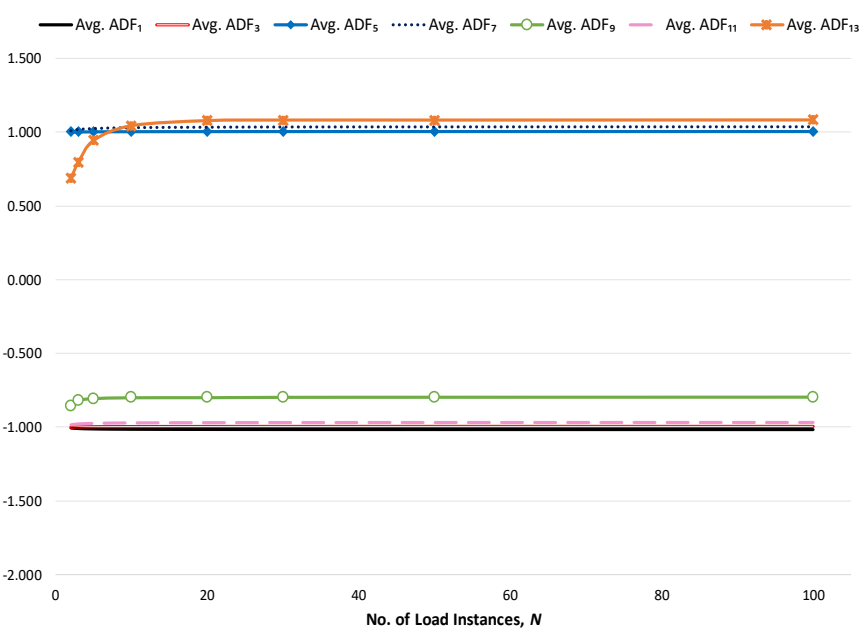

(b)

Figure 11: Mean ADF for harmonic current orders 1, 3, 5, 7, 9, 11 and 13, for number of device instances $N \leq 100$. Mixed lamp type combinations of (a) LED and (b) CFL instances, by harmonic current order

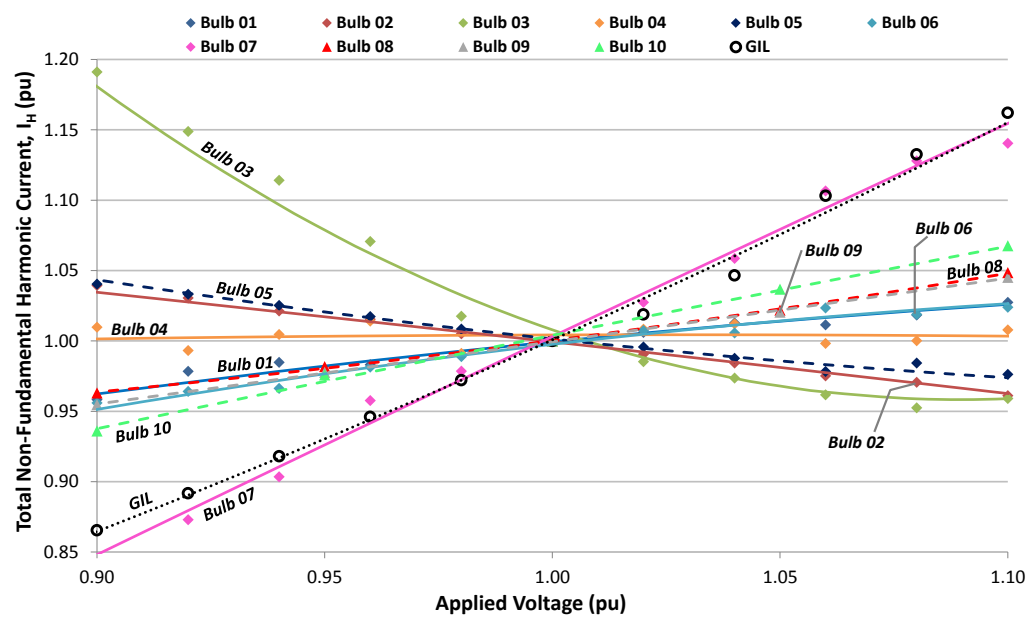

Figure 12: Per-unit total non-fundamental harmonic current vs. voltage; LED, CFL and GIL lamps. 
Table 7: ZIP model representations of $9^{\text {th }}$ order harmonic current, as $\%$ of Fundamental

\begin{tabular}{|c|c|c|c|c|c|c|c|c|c|c|c|c|c|}
\hline \multirow[b]{2}{*}{ Bulb } & \multirow[b]{2}{*}{ Bulb Type } & \multicolumn{5}{|c|}{$\widehat{a_{h}} \equiv\left|\widehat{I_{h}}\right| \cdot \cos \widehat{\phi_{h}}$} & \multicolumn{5}{|c|}{$\widehat{b_{h}} \equiv\left|\widehat{I_{h}}\right| \cdot \sin \widehat{\phi_{h}}$} & \multirow[b]{2}{*}{$\left|\mathbf{I}_{0,9}\right|(\%)$} & \multirow[b]{2}{*}{$\angle \mathbf{I}_{0,9}\left({ }^{\circ}\right)$} \\
\hline & & $\mathbf{a}_{0, h=9}(\%)$ & $\mathbf{R}_{a, 9}^{2}$ & $\mathbf{Z}_{a, 9}$ & $\mathbf{I}_{a, 9}$ & $\mathbf{P}_{a, 9}$ & $\mathbf{b}_{0, h=9}(\%)$ & $\mathbf{R}_{b, 9}^{2}$ & $\mathbf{Z}_{b, 9}$ & $\mathbf{I}_{b, 9}$ & $\mathbf{P}_{b, 9}$ & & \\
\hline 01 & LED/Globe & -35.2 & 1.00 & -3.302 & 8.226 & -3.923 & -4.9 & 1.00 & -13.913 & 10.113 & 4.800 & 35.5 & -172.1 \\
\hline 02 & LED/Globe & -27.2 & 1.00 & -11.085 & 27.762 & -15.676 & -17.7 & 1.00 & -18.275 & 30.924 & -11.649 & 32.4 & -146.9 \\
\hline 03 & LED/Globe & 4.0 & 0.98 & -1.854 & 5.186 & -2.332 & 0.3 & 0.93 & -384.29 & 752.20 & -366.92 & 4.0 & 4.2 \\
\hline 04 & LED/Downlight & 8.4 & 1.00 & -2.318 & 5.885 & -2.568 & -4.4 & 1.00 & -2.696 & 6.634 & -2.939 & 9.5 & -27.5 \\
\hline 05 & LED/Globe & -13.4 & 1.00 & -14.425 & 42.537 & -27.111 & -24.5 & 0.99 & -19.863 & 38.431 & -17.568 & 27.9 & -118.7 \\
\hline 06 & LED/Globe & -8.4 & 1.00 & -5.568 & 7.848 & -1.280 & -6.3 & 1.00 & 1.233 & -0.258 & 0.025 & 10.5 & -143.1 \\
\hline 07 & LED/Downlight & 0.4 & 0.90 & -81.290 & 159.18 & -76.888 & -4.6 & 0.18 & -4.690 & 9.033 & -3.343 & 4.7 & -85.1 \\
\hline 08 & CFL/Spiral & 20.5 & 0.97 & -7.079 & 14.240 & -6.161 & 0.0 & 1.00 & 0.000 & 0.000 & 1.000 & 20.5 & -0.1 \\
\hline 09 & CFL/Triple Tube & 12.9 & 1.00 & -3.441 & 2.424 & 2.016 & -15.9 & 1.00 & -7.439 & 18.087 & -9.648 & 20.5 & -50.9 \\
\hline 10 & CFL/Globe & 18.7 & 1.00 & -1.533 & 2.057 & 0.476 & -8.6 & 1.00 & -7.537 & 20.020 & -11.483 & 20.6 & -24.8 \\
\hline 11 & GIL & 0.0 & - & 0.000 & 0.000 & 1.000 & 0.0 & - & 0.000 & 0.000 & 1.000 & 0.0 & 45.0 \\
\hline
\end{tabular}

Table 8: ZIP model representations of $11^{\text {th }}$ order harmonic current, as $\%$ of Fundamental

\begin{tabular}{|c|c|c|c|c|c|c|c|c|c|c|c|c|c|}
\hline \multirow[b]{2}{*}{ Bulb } & \multirow[b]{2}{*}{ Bulb Type } & \multicolumn{5}{|c|}{$\widehat{a_{h}} \equiv\left|\widehat{I_{h}}\right| \cdot \cos \widehat{\phi_{h}}$} & \multicolumn{5}{|c|}{$\widehat{b_{h}} \equiv\left|\widehat{I_{h}}\right| \cdot \sin \widehat{\phi_{h}}$} & \multirow[b]{2}{*}{$\left|\mathbf{I}_{0,11}\right|(\%)$} & \multirow[b]{2}{*}{$\angle \mathbf{I}_{0,11}\left({ }^{\circ}\right)$} \\
\hline & & $\mathbf{a}_{0, h=11}(\%)$ & $\mathbf{R}_{a, 11}^{2}$ & $\mathbf{Z}_{a, 11}$ & $\mathbf{I}_{a, 11}$ & $\mathbf{P}_{a, 11}$ & $\mathbf{b}_{0, h=11}(\%)$ & $\mathbf{R}_{b, 11}^{2}$ & $\mathbf{Z}_{b, 11}$ & $\mathbf{I}_{b, 11}$ & $\mathbf{P}_{b, 11}$ & & \\
\hline 01 & LED/Globe & 7.0 & 1.00 & -0.912 & 15.985 & -14.072 & 22.6 & 1.00 & -7.362 & 14.484 & -6.122 & 23.7 & 72.7 \\
\hline 02 & LED/Globe & -6.7 & 1.00 & -32.737 & 43.097 & -9.360 & 21.6 & 1.00 & -23.380 & 50.362 & -25.982 & 22.7 & 107.3 \\
\hline 03 & LED/Globe & 3.5 & 0.80 & -2.010 & 4.420 & -1.409 & -0.2 & 0.91 & 487.43 & -948.63 & 462.21 & 3.5 & -2.9 \\
\hline 04 & LED/Downlight & 4.8 & 1.00 & -3.233 & 8.896 & -4.663 & -3.3 & 1.00 & -3.646 & 9.393 & -4.748 & 5.8 & -34.4 \\
\hline 05 & LED/Globe & -18.5 & 1.00 & -27.692 & 50.542 & -21.851 & 13.2 & 1.00 & -30.612 & 72.486 & -40.874 & 22.7 & 144.5 \\
\hline 06 & LED/Globe & -10.1 & 0.99 & -8.254 & 16.533 & -7.280 & -3.9 & 1.00 & 4.479 & -6.827 & 3.348 & 10.8 & -158.6 \\
\hline 07 & LED/Downlight & -0.5 & 0.97 & 70.990 & -125.73 & 55.742 & -3.3 & 0.45 & -3.353 & 6.089 & -1.735 & 3.3 & -98.1 \\
\hline 08 & CFL/Spiral & -0.9 & 1.00 & -109.84 & 302.52 & -191.68 & -17.9 & 0.99 & -11.072 & 23.004 & -10.932 & 17.9 & -92.9 \\
\hline 09 & CFL/Triple Tube & -16.8 & 1.00 & -9.019 & 20.741 & -10.722 & -11.6 & 1.00 & -4.561 & 4.321 & 1.240 & 20.4 & -145.5 \\
\hline 10 & CFL/Globe & -9.3 & 1.00 & -7.781 & 20.117 & -11.336 & -17.3 & 1.00 & -2.548 & 4.276 & -0.728 & 19.6 & -118.2 \\
\hline 11 & GIL & 0.0 & - & 0.000 & 0.000 & 1.000 & 0.0 & - & 0.000 & 0.000 & 1.000 & 0.0 & 45.0 \\
\hline
\end{tabular}

Table 9: ZIP model representations of $13^{\text {th }}$ order harmonic current, as $\%$ of Fundamental

\begin{tabular}{|c|c|c|c|c|c|c|c|c|c|c|c|c|c|}
\hline \multirow[b]{2}{*}{ Bulb } & \multirow[b]{2}{*}{ Bulb Type } & \multicolumn{5}{|c|}{$\widehat{a_{h}} \equiv\left|\widehat{I_{h}}\right| \cdot \cos \widehat{\phi_{h}}$} & \multicolumn{5}{|c|}{$\widehat{b_{h}} \equiv\left|\widehat{I_{h}}\right| \cdot \sin \widehat{\phi_{h}}$} & \multirow[b]{2}{*}{$\left|\mathbf{I}_{0,13}\right|(\%)$} & \multirow[b]{2}{*}{$\angle \mathbf{I}_{0,13}\left({ }^{\circ}\right)$} \\
\hline & & $\mathbf{a}_{0, h=13}(\%)$ & $\mathbf{R}_{a, 13}^{2}$ & $\mathbf{Z}_{a, 13}$ & $\mathbf{I}_{a, 13}$ & $\mathbf{P}_{a, 13}$ & $\mathbf{b}_{0, h=13}(\%)$ & $\mathbf{R}_{b, 13}^{2}$ & $\mathbf{Z}_{b, 13}$ & $\mathbf{I}_{b, 13}$ & $\mathbf{P}_{b, 13}$ & & \\
\hline 01 & LED/Globe & 16.7 & 1.00 & -9.903 & 16.655 & -5.752 & -12.0 & 1.00 & -8.757 & 23.799 & -14.043 & 20.6 & -35.8 \\
\hline 02 & LED/Globe & 21.2 & 0.99 & -30.952 & 62.348 & -30.396 & 0.7 & 1.00 & 0.517 & -231.11 & 231.59 & 21.2 & 1.8 \\
\hline 03 & LED/Globe & 3.1 & 0.83 & -8.291 & 17.412 & -8.121 & -0.4 & 0.87 & 215.38 & -424.42 & 210.04 & 3.1 & -6.7 \\
\hline 04 & LED/Downlight & 2.3 & 1.00 & -3.437 & 11.340 & -6.904 & -2.1 & 1.00 & -1.825 & 6.985 & -4.160 & 3.1 & -42.0 \\
\hline 05 & LED/Globe & 18.0 & 1.00 & -35.654 & 77.569 & -40.915 & 13.8 & 1.00 & -32.129 & 55.419 & -22.291 & 22.6 & 37.5 \\
\hline 06 & LED/Globe & -7.5 & 1.00 & -7.199 & 16.631 & -8.431 & -2.8 & 0.99 & 2.210 & -3.313 & 2.103 & 8.0 & -159.6 \\
\hline 07 & LED/Downlight & -1.4 & 0.97 & 22.487 & -39.107 & 17.619 & -1.9 & 0.58 & 0.419 & -2.736 & 3.317 & 2.4 & -125.9 \\
\hline 08 & CFL/Spiral & -13.2 & 0.99 & -17.836 & 37.247 & -18.411 & -1.2 & 1.00 & 54.211 & -169.84 & 116.63 & 13.3 & -174.7 \\
\hline 09 & CFL/Triple Tube & -6.4 & 1.00 & -7.844 & 6.123 & 2.721 & 15.7 & 1.00 & -11.490 & 25.866 & -13.375 & 17.0 & 112.3 \\
\hline 10 & CFL/Globe & -12.6 & 1.00 & -4.197 & 7.243 & -2.046 & 8.1 & 1.00 & -9.448 & 24.492 & -14.044 & 15.0 & 147.2 \\
\hline 11 & GIL & 0.0 & - & 0.000 & 0.000 & 1.000 & 0.0 & - & 0.000 & 0.000 & 1.000 & 0.0 & 45.0 \\
\hline
\end{tabular}

